\title{
Revisión y análisis panbiogeográfico de las Hymenophyllaceae de las Yungas meridionales de Argentina (Selva Tucumano-Boliviana)
}

Revision and panbiogeographic analysis of Hymenophyllaceae from meridional Yungas from Argentina (Tucumano-Boliviana Rainforest)

\author{
Marcelo D. Arana ${ }^{1,3}$, Cristian Larsen ${ }^{2}$ \& M. Mónica Ponce ${ }^{2}$
}

\begin{abstract}
Resumen
Hymenophyllaceae constituye uno de los linajes más tempranamente divergente dentro de los helechos, habitan lugares hiperhúmedos y su aparición se ha datado en el cretácico temprano, previa a la ruptura de Gondwana; estas características determinan que sean especialmente útiles para establecer patrones biogeográficos. En este trabajo se discuten la diversidad de la familia Hymenophyllaceae en las Yungas meridionales y sus vinculaciones biogeográficas, mediante un análisis panbiogeográfico. Se han encontrado nueve especies, comprendidas en cuatro géneros: Crepidomanes, Didymoglossum, Hymenophyllum y Polyphlebium; Crepidomanes pyxidiferum constituye una novedad florística a nivel genérico para Argentina. Se brindan una clave para todos los géneros de Hymenophyllaceae presentes en Argentina, así como claves entre todas las especies presentes en las Yungas, que además son descriptas e ilustradas.
\end{abstract}

Palabras-clave: Crepidomanes, Dydimoglossum, Hymenophyllum, Polyphlebium, biogeografía.

\begin{abstract}
Hymenophyllaceae represents a very ancient lineage of ferns that inhabit hyperhumid places and appeared in the Early Cretaceous, prior to the breakup of Gondwana; these features make Hymenophyllaceae especially useful to establish biogeographic patterns. The diversity of Hymenophyllaceae in meridional Yungas and their biogeographical relationships, employing a panbiogeographic analysis, are discussed. Nine species in four genera: Crepidomanes, Didymoglossum, Hymenophyllum and Polyphlebium were found; Crepidomanes pyxidiferum constitutes a novelty of generic level for Argentina. A key to all genera of Hymenophyllaceae present in Argentina are provided as well as keys for all species inhabiting the Yungas, which are also described and illustrated. The generalized track shows close relationships among the Argentine Yungas with the North Andes and the Parana dominion, overcoming the arid barrier represented by the Chacoan dominion. Furthermore, the South American Transition Zone constitutes a current barrier between the neotropical and the south-andean Hymenophyllaceae pteridofloras.
\end{abstract}

Key words: Crepidomanes, Dydimoglossum, Hymenophyllum, Polyphlebium, biogeography.

\section{Introducción}

Los Helechos ("monilofitas" sensu Kenrick \& Crane 1997; Pryer et al. 2004) son un grupo monofilético de plantas vasculares que comprende más de 12.000 especies y constituye el segundo grupo más diverso después de las Magnoliidae ("angiospermas"). Dentro del linaje de helechos leptosporangiados, la familia Hymenophyllaceae es un taxón monofilético, tempranamente divergente (Pryer et al. 2004; Smith et al. 2006), cuya primera aparición en el registro fósil se ha datado en el cretácico temprano (Parris 2001; Skog 2001). Se

\footnotetext{
'Universidad Nacional de Río Cuarto, Orientación Plantas Vasculares, Departamento de Ciencias Naturales, Facultad de Ciencias Exactas, Físico-Químicas y Naturales, Ruta 36 km 601, X5804ZAB, Río Cuarto, Córdoba, Argentina.

${ }^{2}$ Academia Nacional de Ciencias Exactas, Físicas y Naturales y CONICET, Instituto de Botánica Darwinion, casilla de correo 22, 1624, San Isidro, Argentina.

${ }^{3}$ Autor para la correspondencia: marana@exa.unrc.edu.ar
} 
caracterizan por presentar láminas extremadamente delgadas, a menudo del grosor de una célula; a su vez carecen por completo de estomas y de epidermis diferenciada, por lo que son plantas altamente dependientes de la humedad ambiental, que habitan solo en lugares hiperhúmedos.

La familia Hymenophyllaceae se encuentra diversificada ampliamente, tanto en la morfología (rizomas erectos o rastreros, raíces robustas o sin raíces, hojas enteras a muy divididas, etc.) como en el hábito de crecimiento, que puede ser terrestre, epipétrico, epífito o escandente (Dubuisson et al. 2003a). En conjunto, por su antigüedad, monofilia, estasis morfológico y las particularidades adaptativas de los modos de dispersión de esta familia y en general de los helechos, éstos son especialmente apropiados para establecer patrones biogeográficos (Wolf et al. 2001; Moran 2008; Kessler 2010; Sanguinés-Franco et al. 2011; Arana et al. 2013).

Los helechos en general son plantas extremadamente vulnerables a la extinción, debido a la permanente fragmentación y deforestación de los bosques montanos tropicales y templados que ocurre actualmente, donde se encuentran los nichos ecológicos más favorables para estos taxones y alcanzan la mayor diversidad (Arcand \& Ranker 2008). En los estratos arbóreos de zonas montañosas húmedas, los helechos y licofitas juegan un rol importante en el balance hídrico (Ambrose 2004) y en la estructura de la vegetación (Hill \& Silander 2001; Paciencia \& Prado 2005).

Las Yungas constituyen una de las regiones caracterizada por este tipo de bosques o selvas montanos húmedos, y comprenden una angosta faja en las laderas orientales de los Andes, entre los 300 y los 2500 a $3500 \mathrm{~m}$ de altitud, desde Venezuela hasta el noroeste de la Argentina (Cabrera \& Willink 1980). El sector meridional de aquella extensa provincia biogeográfica, denominado Selva Tucumano-Boliviana (o Boliviano-Tucumana, sensu Navarro \& Maldonado 2002) comprende el área al sur de los $18^{\circ} \mathrm{S} 64^{\circ} 30 \mathrm{~W}$, es decir al sur del "codo andino", y es considerada por muchos autores como una unidad fitogeográfica diferente, definida por Hauman (1931) y utilizada por la mayoría de los autores posteriores (Castellanos \& Perez-Moreau 1944; Parodi 1945). Para los helechos y licofitas, esta última región constituye uno de los tres centros de concentración del Cono Sur (de la Sota 1973; Ponce et al. 2002), con alrededor de 200 especies (Ponce \& de la Sota 2008; Larsen et al. 2010; Arana et al. 2012; Ganem et al. 2013).
En las Yungas meridionales son particularmente escasos los estudios de la vegetación en superficies de una hectárea o más, ya que la gran diversidad biológica existente, sumada a la variabilidad altitudinal y latitudinal y a la dificultad de acceso y desplazamiento en la región, han limitado los estudios de numerosos aspectos de su funcionamiento y de su composición florística (Zenteno-Ruiz \& López 2010; Ganem et al. 2013). Además, la biogeografía de las Yungas no es del todo clara, ya que hay afinidades andinas por un lado, y paranaenses por el otro (Zenteno-Ruiz \& López 2010; Arana et al. 2012). Por eso algunos autores ubican a la Yungas y al bosque Paranaense en dominios separados (Dominio Suroeste Amazónico, y Paraná respectivamente, sensu Morrone 2014), mientras otros como por ejemplo Cabrera \& Willink (1980) las agrupan dentro de un solo dominio, el Amazónico. Debido a que Hymenophyllaceae es un taxón monofilético muy antiguo, y uno de los grupos de helechos mejor representados en la región de las Yungas, los objetivos de este trabajo son presentar una actualización sistemática de la familia en esta región y establecer sus vinculaciones biogeográficas mediante un análisis panbiogeográfico.

\section{Materiales y Métodos}

Área de estudio

Las Yungas meridionales, o selvas TucumanoBolivianas se extienden desde los departamentos de Tarija, Chuquisaca y Santa Cruz en Bolivia hasta las provincias de Tucumán y Catamarca en la Argentina, conformando una sola unidad desde punto de vista biogeográfico, ecológico y social (Grau \& Brown 2000; Brown et al. 2001; Kessler \& Beck 2001; Navarro \& Maldonado 2002).

El presente trabajo se centra en las Yungas de Argentina que ocupan una superficie estimada actual de 5,2 millones de has, extendiéndose desde la frontera con Bolivia $\left(23^{\circ} \mathrm{S}\right)$ hasta el norte de la provincia de Catamarca $\left(29^{\circ} \mathrm{S}\right)$, abarcando las Provincias de Salta, Jujuy y Tucumán (Fig. 1). Presentan una longitud de $600 \mathrm{~km}$ en sentido NorteSur y menos de $100 \mathrm{~km}$ de ancho, en un rango altitudinal entre los 400 y $3000 \mathrm{~m}$ s.m. (Brown et al. 2001, 2006). El clima es cálido y húmedo con lluvias estivales, con heladas y nevadas frecuentes en las zonas más elevadas. Las Yungas en la Argentina presentan además un gradiente latitudinal de diversidad biológica originado principalmente por la discontinuidad de las masas de bosques que, a su vez, es producto de la irregular distribución de los cordones montañosos donde se sitúan. En 


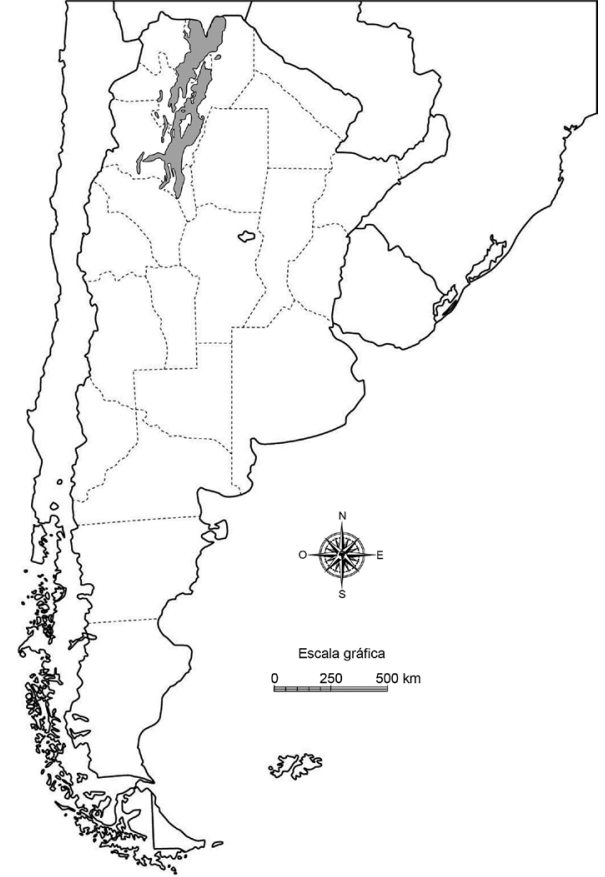

Figura 1 - Área de estudio. La zona gris representa la ubicación y los límites de las Yungas en Argentina. Figure 1 - Study area. The gray area represents the location and boundaries of the Yungas in Argentina.

tal sentido, se reconocen tres sectores geográficos latitudinales (norte, centro y sur), que coinciden con los grandes bloques orográficos y que se conectan entre sí a través de los bosques chaqueños serranos en las áreas intermedias (Brown et al. 2002).

A lo largo del gradiente altitudinal se reconocen esquemáticamente tres pisos de vegetación con características fisionómicas y florísticas diferenciables: (i) Selva Pedemontana, ocupa los sectores entre los 400 y $700 \mathrm{~m}$ s.m. en el pedemonte y las serranías de escasa altitud. (ii) Selva Montana, ocupa las laderas de las montañas entre los 700 y los $1.500 \mathrm{~m}$ s.m., y es un bosque con predominio de especies perennifolias y con una estacionalidad hídrica menos marcada que la Selva Pedemontana. Las especies dominantes son de origen tropical y presentan en esta región su límite meridional de distribución geográfica; y (iii) Bosque Montano, entre los 1.500 y 3.000 m s.m., que muestra el paisaje con mayor heterogeneidad estructural dada por bosques en distintos estadíos sucesionales. En este tipo de ambientes, en el gradiente comprendido entre los 600 y los $2100 \mathrm{~m}$ s.m. han sido colectadas las Hymenophyllaceae para esta área.

En conjunto, las Yungas y la Selva Misionera del noreste de Argentina (Dominio Parana sensu
Morrone 2014; Provincia Paranaense sensu Cabrera \& Willink 1980) representan menos del 2\% de la superficie de Argentina continental, pero acumulan más del 50\% de la biodiversidad del país (Brown et al. 2002).

\section{Análisis taxonómico}

El estudio se llevó a cabo utilizando material colectado durante diferentes viajes de campo durante los años 2010-2015, la consulta bibliográfíca y revisión de los ejemplares depositados en los siguientes herbarios (Thiers, continuamente atualizado): BA, BAB, CORD, CTES, JUA, MCNS, MERL, LIL, LP, LPB, RCVC, RIOC y SI.

En este trabajo se sigue el tratamiento filogenético de las Hymenophyllaceae propuesto por Ebihara et al. (2006) y Dubuisson et al. (2013) que delimitan la familia en nueve géneros y 600 especies. Para el análisis morfológico de los taxones locales se tomaron en cuenta caracteres como la longitud del rizoma; la densidad y el color de los pelos del rizoma; la presencia o ausencia, densidad y grosor de las raíces; la venación de sus hojas (catádroma o anádroma); la presencia de falsas venillas en las láminas; el grado de división de las láminas; la simetría de las pinnas; la presencia o ausencia de tricomas en el margen de las láminas; la presencia o no de pelos sobre pecíolos y raquis, el tipo de involucro, bivalvo o tubular, y el grosor de la lámina (Larsen et al. 2013). Se elaboró una clave general para identificar los géneros presentes en todo el territorio argentino y, dentro de cada género, claves para las especies presentes en las Yungas. Para cada entidad se proporciona descripción, ilustraciones, distribución geográfica y datos del hábitat en el área de estudio. En cuanto a la nomenclatura, sólo se mencionan los basónimos y sinónimos relevantes para el área de estudio; una lista completa de los sinónimos se puede consultar en Ponce \& de la Sota (2008).

\section{Análisis biogeográfico}

Para establecer la dimensión espacial (geográfica) de la diversidad de las Hymenophyllaceae se aplicaron los conceptos de la panbiogeografía (Morrone 2009). El análisis consistió en marcar en mapas los puntos de distribución de las distintas especies para luego unir estas localidades de registro mediante líneas con el criterio de distancia mínima, denominadas trazos individuales (Morrone \& Crisci 1995; Espinosa et al. 2002; Zunino \& Zullini 2003; Morrone 2004). Una vez obtenidos los trazos individuales, se superpusieron para establecer la existencia de los trazos generalizados, 
que permiten establecer la existencia de una biota ancestral distribuida ampliamente y fragmentada por eventos vicariantes y constituyen la expresión geográfica de componentes bióticos (Morrone 2009). La convergencia de dos o más trazos generalizados en un área determinada conforma un nodo panbiogeográfico, sitio que puede ser importante o prioritario para la conservación (Espinosa et al. 2002; Morrone 2004, 2009; Arana et al. 2013; Talonia \& Escalante 2013).

\section{Resultados y Discusión}

\section{Tratamiento taxonómico}

Hymenophyllaceae Gaudich., Freyc. Voy. Bot.: 262. 1826. TIPO: Hymenophyllum Sm. Hymenophyllaceae Link, Handb. Z. Erk. d. Gew. 3: 36. 1833. Hymenophyllaceae Mart., Consp. Regn. Veg. 3. Sep-Oct 1835 (como "Hymenophylleae"). Trichomanaceae Burmeist., Handb. Naturgesch.: 196. 1836. Trichomanaceae Kunkel, Feddes Rep. 70: 155.1965.

Plantas con rizomas rastreros, pocas veces erectos, pilosos. Hojas uniformes o dimórficas. Láminas enteras o divididas, lineales, ovadas a flabeladas, constituidas por uno o pocos estratos de celulas, sin estomas, epidermis no diferenciada, cutícula ausente o muy reducida; glabras o con pelos en los márgenes, ocasionalmente en la superficie. Soros marginales, en el ápice de las venas, con indusio cónico, campanulado, tubular o valvar, con receptáculos inclusos o exertos. Esporangios con un anillo oblicuo ininterrumpido. Esporas verdes, con clorofila, globosas, triletes. Gametofitos verdes epígeos, filamentosos o acintados, a veces con multiplicación por gemación o fragmentación y, en algunos casos, persistentes indefinidamente, sin producir esporofitos.

Comprende nueve géneros y alrededor de 600 especies de distribución pantropical y templado-fría de ambos hemisferios (Ebihara et al. 2006). En Argentina se encuentran seis géneros: Crepidomanes, Didymoglossum, Hymenophyllum, Polyphlebium, Trichomanes y Vandenboschia con 32 especies. El género más grande es Hymenophyllum con 22, encontrándose la mayoría de las especies (19) en los bosques templados (Larsen et al. 2013). En la región estudiada se encuentran nueve especies reunidas en cuatro géneros (Crepidomanes, Didymoglossum, Hymenophyllum y Polyphlebium), con un género y una especie citados por primera para el país. Trichomanes y Vandenboschia (con tres y una especie respectivamente) no se tratan en este trabajo porque se hallan en la selva paranaense de la provincia política de Misiones, fuera del área de este estudio.

\section{Clave para la identificación de los géneros de Argentina}

1. Soros con involucro bivalvado 3. Hymenophyllum

1'. Soros con involucro tubular u obcónico, no bivalvado

2. Plantas con raíces verdaderas.

3. Rizomas con escasos pelos castaño claros, raíces pocas y delgadas

4. Polyphlebium

3'. Rizomas densamente cubierto de pelos castaños, castaño brillantes o negruzcos, raíces numerosas y robustas.

4. Pinnas simétricas

Trichomanes

4'. Pinnas asimétricas

Vandenboschia

2'. Plantas sin raíces, a veces con ejes similares a raíces, cubiertos densamente por pelos negros, al igual que el rizoma.

5. Láminas con venillas falsas continuas y paralelas a las venas.

2. Didymoglossum

5'. Láminas sin falsas venillas.

1. Crepidomanes

Descripción de los taxones

1. Crepidomanes pyxidiferum (L.) Dubuisson

\& Ebihara, Acta Bot. Gall. 160 (2): 186.

Trichomanes pyxidiferum L., Sp. Pl. 2: 1098. 1753.

Vandenboschia pyxidifera (L.) Copel., Philipp. J.

Sci. 67: 53. 1938. Polyphlebium pyxidiferum (L.)

Ebihara \& Dubuisson, Blumea 51: 240-241, 2006.
TIPO: 'Habitat in America', Plumier, Traité Foug. Am. fig. 50 E, 1705!, (Lectotipo designado por Proctor, 1985: 106).

Fig. 2a-b

Plantas con rizomas rastreros, ramificados, delgados, filiformes, de aprox. 0,4-0,5 $\mathrm{mm}$ de diámetro, densamente cubierto con pelos negruzcos. Hojas remotas de hasta $13 \times 3 \mathrm{~cm}$. 
Pecíolos teretes o aplanados, con alas de borde generalmente plano o raras veces ondulado, hasta de $3 \mathrm{~cm}$ long., glabros o con pelos diminutos en el lado abaxial, con la base cubierta por pelos del mismo tipo que el rizoma. Láminas de contorno ovado a elíptico-lanceolado, simétricas a ambos lados del raquis, 2-3-pinnatífidas, glabras, abrupta o gradualmente reducida hacia la base, venación anádroma. Raquis primario alado, con alas de borde plano o levemente ondulado. Pinnas remotas, de base adnata al raquis primario y con raquis secundario alado. Segmentos ascendentes, no superpuestos, oblongos a lineales, con pliegues longitudinales paralelos a la única vena, márgenes planos, ápice bífido o emarginado. Soros subaxilares, en las pinnas basales y comúnmente uno por pinna, con involucros cónicos, alados, las alas de tres o cuatro hileras de células de ancho, cónicos, 1-1,5 veces tan largo como ancho, con labios muy levemente diferenciados. Receptáculo largamente exerto, filiforme, con numerosos esporangios.

El género Crepidomanes se distribuye principalmente en los trópicos del viejo mundo, con una especie neotropical (Dubuisson et al. 2013), presente en Antillas, América Central y del Sur, donde se la encuentra en Colombia, Venezuela, Guayanas, Ecuador, Perú, Brasil, Bolivia y ahora en la provincia de Salta, Argentina, límite austral de distribución. Es una especie epífita, común en la selva basal.

Crepidomanes pyxidiferum fue recientemente transferida al género Crepidomanes basándose en estudios de filogenia molecular (Dubuisson et al. 2013). Morfológicamente se caracteriza por el pecíolo y raquis con alas de borde ondulado o plano. De acuerdo a Tryon \& Stolze (1989) es la única especie que posee pliegues longitudinales paralelos a las venas en los últimos segmentos. Es muy cercana a las especies paleotropicales
C. melanotrichum (Schltdl.) J.P. Roux y $C$. inopinatum (Pic. Serm.) J.P. Roux. En el Neotrópico puede confundirse con especies del género Polyphlebium que poseen raíces (mientras que en Crepidomanes las raíces están ausentes); entre ellas Polyphlebium hymenophylloides (Bosch) Ebihara \& Dubuisson y P. borbonicum (Bosch) Ebihara \& Dubuisson. Estas últimas habitan en Bolivia y no alcanzarían a Argentina en su distribución sur. Además C. pyxidiferum puede diferenciarse de $P$. hymenophylloides y $P$. borbonicum por la forma de los soros cuyos involucros son dos o tres (a veces más) más largos que anchos.

Material seleccionado: ARGENTINA, SALTA, Dpto. Orán, Aguas Blancas, finca El Arrazayal, 600 m, 16.III.1986, C. Palaci 618 (MCNS).

2. Didymoglossum Desv., Mém. Soc. Linn. Paris 6: 330. Jul 1827.Trichomanes L. sect. Didymoglossum (Desv.) T. Moore, Index Fil. cx. 1857. TIPO: Didymoglossum muscoides (Sw.) Desv. $=$ Didymoglossum hymenoides (Hedw.) Copel. (Lectotipo elegido por Christensen, Index Fil. 14. 1906, como Trichomanes hymenoides Hedw.).

Rizomas largamente rastreros, filiformes, cubiertos por pelos oscuros, densamente dispuestos, sin raíces o con brotes como raíces. Hojas distanciadas, de 1-6 cm de largo. Láminas ovadas hasta elípticas, flabeladas, lobadas o pinnatífidas, hasta pinnadas, de márgenes enteros, glabros o con pelos; con varias venas y falsas venillas, paralelas a las venas verdaderas. Soros a menudo inmersos en la lámina, campanulados a tubulares, bilabiados de margen castaño oscuro, con receptáculo exerto.

Más de 30 especies de las regiones tropicales, de pequeño tamaño y hábito epifítico o epilítico (Wessels Boer 1962). En Argentina se encuentran 3 especies que habitan en las Yungas $\mathrm{y}$ en Misiones.

\section{Clave para la identificación de las especies de Didymoglossum de Yungas argentinas}

1. Láminas pinnatífidas a bipinnatífidas; soros parcialmente inmersos en el tejido foliar, o al menos alados 2.2. D. krausii

1'. Láminas lobadas a pinnatisectas, a veces flabeliformes; soros no inmersos en el tejido foliar, ni alados.

2. Venillas falsas muy escasas, labios del indusio mucho más anchos que largos; la mayoría de las células del tejido foliar isodiamétricas.

2. Venillas falsas abundantes, labios del indusio tan largos, o más, como anchos; la mayoría de las células del tejido foliar elongadas 

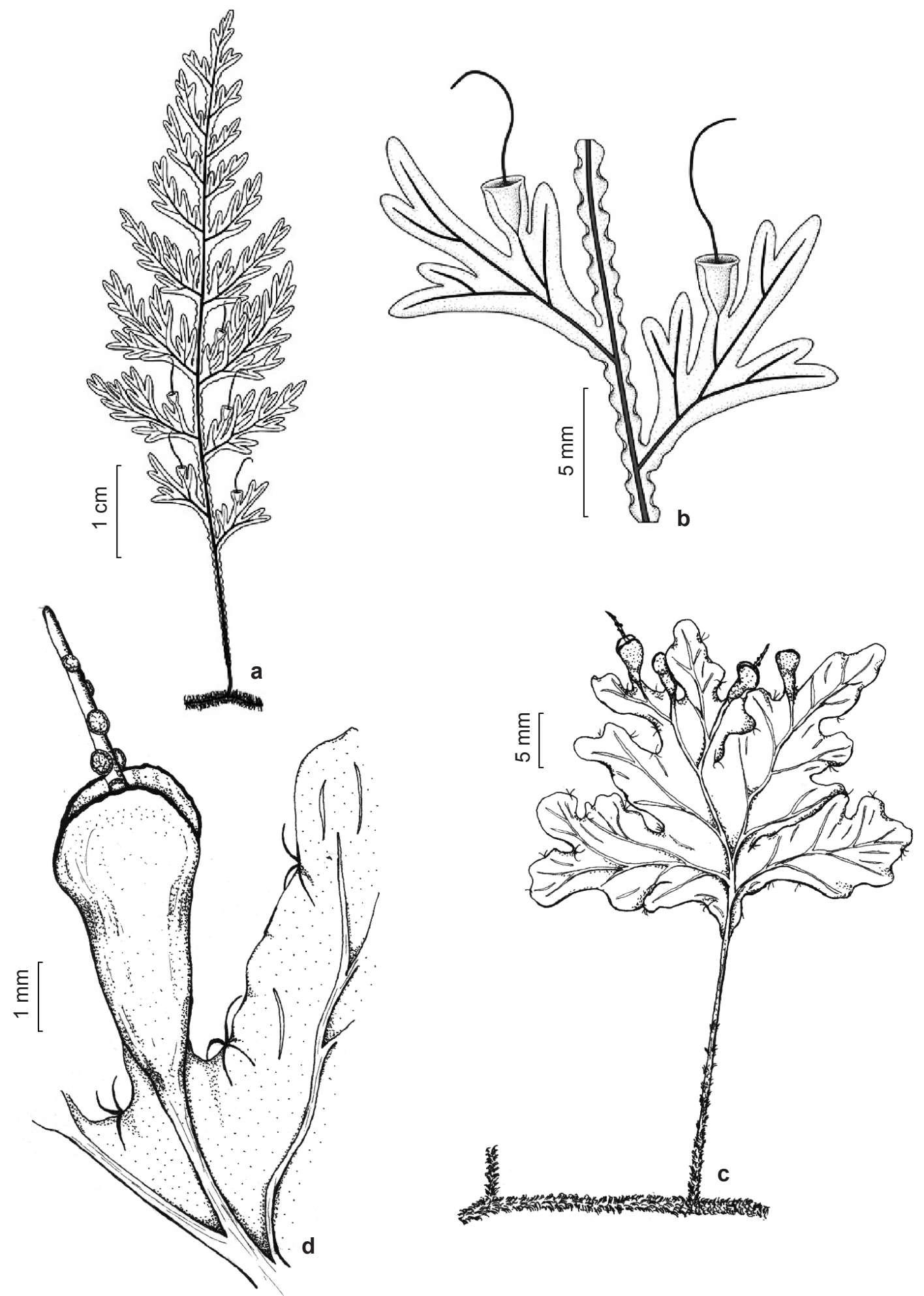

Figura 2 -a-b. Crepidomanes pyxidiferum - a. aspecto general; b. detalle de un par de pinnas mostrandoposición de los soros. (Palací 618 - MCNS). c-d. Didymoglossum hymenoides - c. aspecto general; d. detalle de un soro. (modificado de E.R. de la Sota 1977). Figure 2 - a-b. Crepidomanes pyxidiferum - a. habit; b. detail of a pair of pinnae showing sori position. c-d. Didymoglossum hymenoides - c. habit; d. detail of a sorus (modified from E.R. de la Sota 1977). 
2.1. Didymoglossum hymenoides (Hedw.) Copel., Philipp. J. Sci. 67: 77. 1938. Trichomanes hymenoides Hedw., Fil. Gen. Sp.: t. 3, f. 3. 1799. TIPO: Hedwig, Fil. Gen. Sp. t. 3, f. 3, 1799. (Lectotipo designado por W. Boer, Acta Bot. Neerl. 11: 306. 1962, probablemente basado en el ejemplar: Jamaica, O. Swartz s.n., BM000936817!, P00623918!).

Fig. 2c-d

Rizomas largamente rastreros, cubiertos por pelos negruzcos. Hojas remotas, de (0,5-)1-3,5 $\times 1,5 \mathrm{~cm}$; subsésiles o cortamente pecioladas. Pecíolos con la base cubierta por las mismas escamas que los rizomas. Láminas ovadas a elípticas, las jóvenes a menudo subreniformes, pinnatífidas o pinnatífidas con pinnas lobuladas, con tricomas oscuros, simples a bífidos sobre el margen, y estrellados en los senos foliares; retículo celular de la lámina con células isodiamétricas; falsas venillas escasas, situadas entre las venas verdaderas. Soros ubicados próximos al ápice de la lámina, notablemente exertos, con sólo la base inmersa en el tejido fotosintético; indusio no alado, o con un ala muy estrecha; cónico, bilabiado; usualmente más ancho que largo; receptáculo notablemente exerto.

Esta especie se distribuye en América Central desde el sur de México y Panamá, y Sudamérica desde Venezuela y Colombia hasta Uruguay y Argentina, en las provincias de Jujuy, Misiones y Tucumán (Davidse et al. 1995). Viven en las orillas de ríos o cascadas, son epífitos o epipétricos. Material seleccionado: ARGENTINA, TUCUMÁN, Dpto. Chicligasta, Alpachiri, cruzando el río Sonador, camino a Casa de Piedra, 840 m, 03.XI.2009, C. Larsen \& E. Ulibarri 79 (SI); desde Casa de Piedra hacia Alpachiri, 04.XI.2009, C. Larsen \& E. Ulibarri 112 (SI); Arroyo Celeste, H. Fabris 6606 (LP).

2.2. Didymoglossum krausii (Hook. \& Grev.) C. Presl, Abh. Böhm. Ges. Wiss. 5: 115. 1843. Trichomanes krausii Hook. \& Grev., Ic. Fil. t. 149. 1831. TIPO: Dominica, Kraus s.n. (Holotipo E00205306!).

Fig. 3a-b

Rizomas largamente rastreros, densamente cubiertos por pelos negruzcos. Hojas remotas, 2-8 $\mathrm{cm}$, subsésiles o cortamente pecioladas. Pecíolos de $0,5-1,4$ cm long., con la base cubierta por los mismos pelos que el rizoma. Láminas de contorno elíptico, ovado u obovado, 1-2-pinnatífidas, de $2-3 \times 1-1,6 \mathrm{~cm}$, con el raquis bien diferenciado, a menudo con pelos oscuros en la cara abaxial, al menos cerca de la base; con pelos simples y bífidos en el margen foliar, estrellados en los senos foliares o sobre dientes. Segmentos angostos, venillas falsas muy escasas, distantes y subparalelas al margen. Soros terminales sobre los segmentos de la parte apical de la lámina, uno a varios por lámina; parcial o profundamente inmersos en ella, la porción exerta alada. Indusios cónicos, bilabiados, con labios más anchos que el tubo, de márgenes oscuros. Receptáculo exerto.

Esta especie se caracteriza por tener los pecíolos y la cara abaxial del raquis cubiertos por pelos castaño oscuros, mientras que en las especies cercanas son esencialmente glabras (Tryon \& Stolze 1993). Se encuentra desde Florida (EUA), Méjico y Antillas hasta Paraguay y Argentina (Davidse et al. 1995), en las provincias de Misiones y Salta (Ponce \& de la Sota 2008). Crece sobre piedras, entre musgos, en borde de arroyos o base de troncos.

Material seleccionado: ARGENTINA, SALTA, Dpto. Orán, Urundel, confluencia de los ríos Santa María y Santa Rosa, R. Capurro 256 (LP).

2.3. Didymoglossum reptans (Sw.) C. Presl, Hymenophyllaceae 23. 1843. Trichomanes reptans Sw., Prodr.: 136. 1788. TIPO: Jamaica, Swartz s.n. (Holotipo SBT 10575!). Fig. 3c-d

Plantas con rizomas delgados, rastreros, con una densa cobertura de pelos oscuros. Hojas remotas, de 2-9 cm, subsésiles o cortamente pecioladas. Pecíolos con pelos iguales a los del rizoma en su base, angostamente alados hacia el ápice. Lámina de contorno elíptico a elípticolanceolado, con la base atenuada y la parte superior ensanchada, de 2,5-4,5 × 1-3 cm, pinnatífida a pinnatisecta. Segmentos enteros a pinnatífidos, con numerosas venillas falsas que no se disponen paralelas al margen, extendiéndose hacia el margen de la lámina; retículo del tejido fotosintético con la mayoría de las células elongadas; margen con pelos simples a bifurcados, castaño oscuros, estrellados en los senos foliares. Soros agrupados en el extremo apical de la lámina, involucros libres o solo la parte basal inmersa, angostamente cónicos, bilabiados, con labios de márgenes oscuros y curvados a la madurez. Receptáculos exertos.

Plantas epifíticas o epilíticas que habitan desde el sur de México, Antillas, Venezuela, Colombia, Ecuador, Perú, Brasil y Argentina, en las provincias de Misiones, Jujuy y Tucumán (Ponce $\&$ de la Sota 2008). En el Noroeste de Argentina esta especie crece sobre rocas en cavernas húmedas y sombrías, bordes de arroyos y en la base de los troncos. 

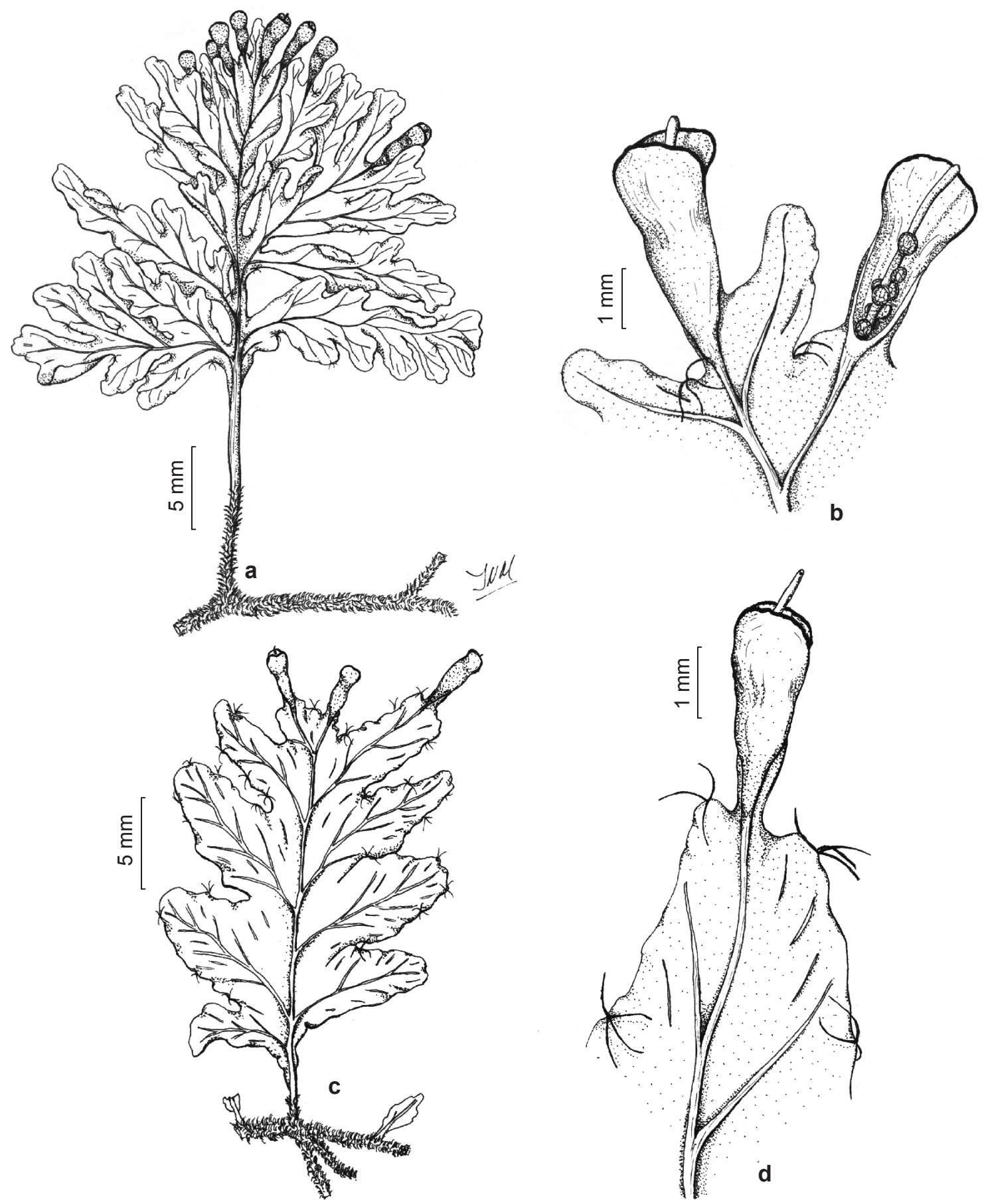

Figura 3 - a-b. Didymoglossum krausii - a. aspecto general; b. detalle de los soros. (modificado de E.R. de la Sota 1977). c-d. Didymoglossum reptans - c. aspecto general; d. detalle de un soro. (modificado de E.R. de la Sota 1977). Figure 3 - a-b. Didymoglossum krausii - a. habit; b. detail of sori (modified from E.R. de la Sota 1977). c-d. Didymoglossum reptans - c. habit; d. detail of a sorus (modified from E.R. de la Sota 1977). 
Material seleccionado: ARGENTINA, JUJUY, Dpto. Ledesma, Parque Nacional Calilegua, $1600 \mathrm{~m}$, 24.VIII.2010, O. Martínez \& R. Guerra 1987 (SI), cerca de Agua del Tigre, $1630 \mathrm{~m}, 05 . \mathrm{VII} .2010, C$. Larsen \& M. Arana 161 (SI, RCVC); Idem, Aguada de Tigre, 25.V.2011, A. Ganem et al. 44 (JUA, LP, RCVC). TUCUMÁN, Dpto. Chicligasta, Alpachiri, río Cochuna, 14.X.1966, A. Burkart 26540 (SI), Monteros, La Quebrada, II.1912, L. Castillón (SI 21716).

3. Hymenophyllum Sm., Mém. Acad. Turin 5: 148. 1793. TIPO: Hymenophyllum tunbrigense (L.) Sm., designado por C. Presl, 1843: 31.

Plantas epífitas, epipétricas o raramente terrestres; rizomas largamente rastreros, pilosos, los tricomas castaños o rojizos, simples, 2-pluricelulares; hojas monomórficas, delicadas, en general pequeñas, hasta de $60 \mathrm{~cm}$, distantes; pecíolos generalmente más cortos que la lámina, teretes, sin alas o alados, glabros a pilosos; láminas simples o lobadas a varias veces divididas, determinadas o indeterminadas, lineales a subflabeladas, planas, onduladas o crespadas, glabras a densamente pilosas o solo con pelos sobre las venas y/o márgenes, los pelos simples, bifurcados o estrellados; últimos segmentos lineales, con los márgenes enteros a aserrados con una sola vena; venación libre, anádroma, generalmente pinnada o subflabelada, sin venillas falsas; soros marginales con involucro 2-valvado, parcialmente inmerso en la lámina a ligeramente pedunculado, orbiculares a elípticos u obovados; esporangios sobre un receptáculo filiforme a engrosado, cilíndrico a angostamente turbinado (obcónico), rudimentario o corto, hasta algo exerto en la madurez.

\section{Clave para la identificación de las especies de Hymenophyllum de Yungas argentinas}

1. Láminas glabras, ocasionalmente con pelos rojizos simples sobre los ejes.

2. Márgenes de los segmentos dentados. Soros en general uno por pinna. Pelos rojizos sobre los ejes 3.4. H. tunbrigense var. cordobense

2'. Márgenes de los segmentos enteros. Soros, más de uno por pinna. Ejes glabros 3.3. H. polyanthos

1'. Láminas con pelos bifurcados o estrellados sobre venas y márgenes.

3. Láminas ondulado-crespadas. Pelos de lós márgenes simples o bifurcados. 3.2. H. crispum

3'. Láminas planas. Pelos de lós márgenes en general doblemente estrellados desde la base, en ocasiones estrellados o bifurcados. 3.1. H. capurroi

3.1. Hymenophyllum capurroi de la Sota, Darwiniana 17: 54, f. 5a-c, pl. 1, 2. 1972. TIPO: Argentina, Tucumán, Chicligasta, Alpachiri, Arroyo Las Pavas, R. Capurro 105 (Holotipo LP!; Isotipos BA!, LIL!, US imagen!). $\quad$ Fig. 6a-e

Rizomas rastreros, filiformes, con pocos pelos, de 0,12-0,17 $\mathrm{mm}$ de diámetro. Hojas remotas, de 3,5-13,5 cm long. Pecíolos capilares, de $0,3-3,5 \mathrm{~cm}$, no alados, con escasos pelos simples, traslúcidos, raramente bifurcados o estrellados. Láminas de contorno ovadolanceolado u ovado-lineal-lanceolado, pinnatífidas a 2-3-pinnatífidas, delicadas, de 3,3-11 × 1-1,9 $\mathrm{cm}$. Raquis anchamente alado. Pinnas hasta 8 pares por lámina, ascendentes, anchamente adnatas, simples o con 2-4 lacinias, raro 5, las basales distantes y reducidas. Segmentos lineares, con ápice redondeado a un poco ensanchado, de 1,5-2 $\mathrm{mm}$ de ancho, uninervios, planos, densamente pilosos sobre la vena y márgenes; pelos en general biestrellados, a veces agrupados, con 3-4 ramas, largamente estipitados. Soros en el extremo de segmentos de largo normal, en general sobre las pinnas medias y apicales. Involucros orbicular-cuneados, en general un poco más angostos que los segmentos, densamente cubiertos con pelos biestrellados. Receptáculos filiformes, totalmente insertos.

Crece en Bolivia y en Argentina, en Tucumán, Salta y Jujuy (Ponce \& de la Sota 2008). Se halla como epífito o epipétrico en la selva a partir de los $1200 \mathrm{~m}$ s.m.

Material seleccionado: ARGENTINA, JUJUY, Depto. Ledesma, Parque Nacional Calilegua, 1500 m, 26.X.2007, Martínez 1428 (MCNS); descendiendo desde Monolito hacia Mesada de las Colmenas, $1616 \mathrm{~m}$, 05.VII.2010, C. Larsen \& M. Arana 138 (SI, RCVC); Aguada de Tigre, II.2009, Ramos Giacosa et al. 30 (JUA, LP). SALTA, Depto. Santa Victoria, Parque Nacional Baritú, Ayo. Baritú, bosque de Podocarpus sp., 16001700 m, 4.X.1985, A. Brown 2022 (SI). TUCUMÁN, R.H. Capurro 105 (LIL); Depto. Chicligasta, Casa de Piedra, afluente del arroyo del Bajo Grande, $1233 \mathrm{~m}$, 04.XI.2009, C. Larsen \& E. Ulibarri 108 (SI). 
3.2. Hymenophyllum crispum Kunth, Nov. Gen. Sp. 1: 26. 1815. TIPO: Venezuela, Silla de Caracas, Humboldt \& Bonpland s.n. (Holotipo B 200095682 !; Isotipo P00622086!). Fig. 4a-e

Rizomas filiformes, rastreros, ramificados, parduzcos, $0,25 \mathrm{~mm}$ de diámetro, con pelos simples, septados. Hojas de 5-10 cm long., distantes. Pecíolos 2,1-4 cm, teretes, con pelos simples y bifurcados, alados en el ápice. Láminas de $2,5-8 \times 1,5-2,6 \mathrm{~cm}$, lineares a elíptico-lanceoladas, 2-pinnatífidas, con pelos bifurcados o estrellados sobre las venas, y simples o raramente bifurcados

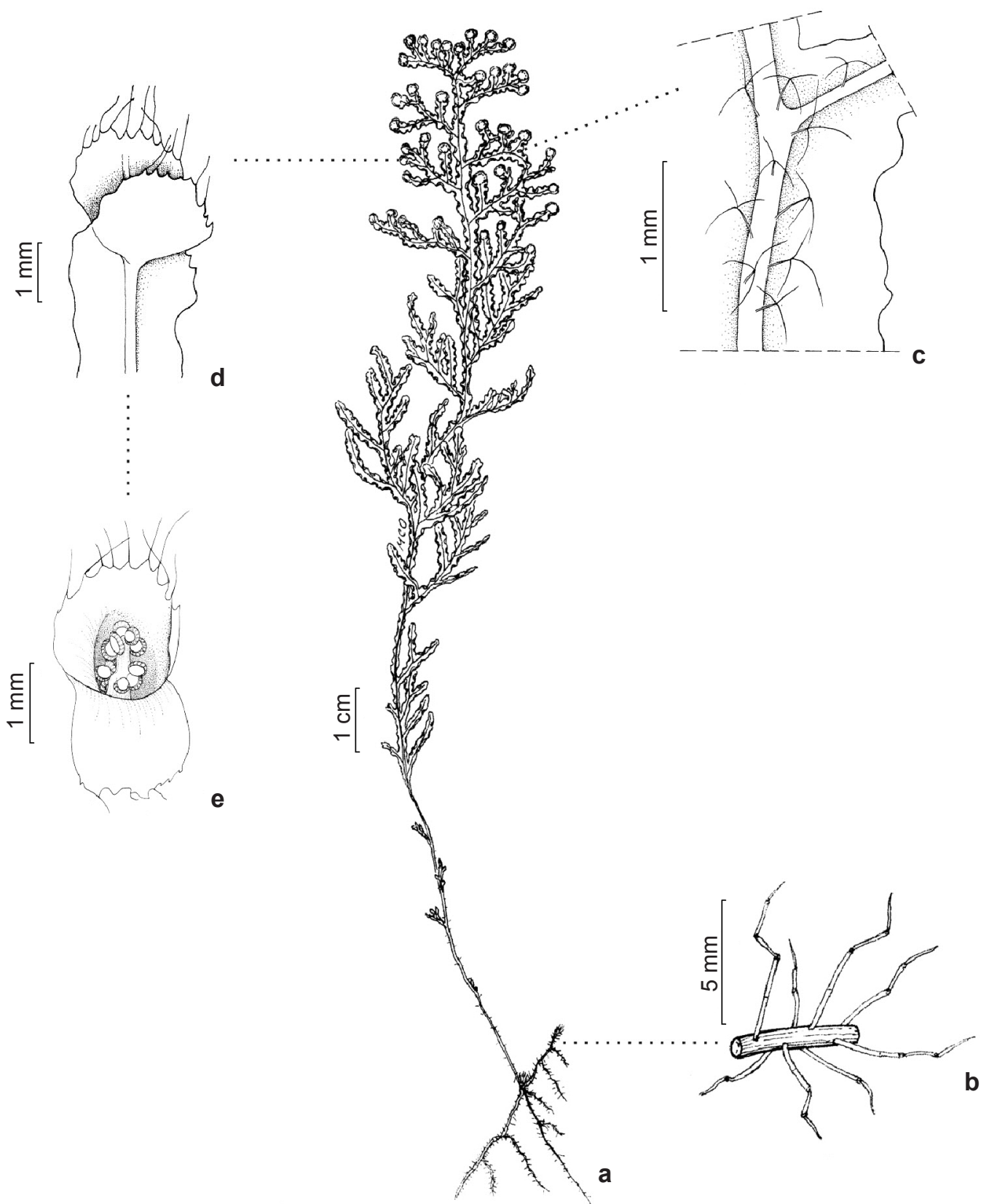

Figura 4 - a-e. Hymenophyllum crispum - a. aspecto general; b. aspecto del rizoma, mostrando pelos septados; c. detalle del raquis con pelos estrellados; d. detalle de un soro, mostrando los pelos del borde; e. soro abierto mostrando receptáculo y esporangios. (Martínez \& Chambi 1780 - MCNS).

Figure 4 - a-e. Hymenophyllum crispum - a. habit; b. rhizome showing septate hairs; c. detail of the rachis with stellate hairs; d. detail of a sorus showing the hairs of the margin; e. open sorus showing receptacle and sporangia. (Martínez \& Chambi 1780 - MCNS). 
en los márgenes, la base reducida, el ápice irregular. Raquis alado, castaño, con pelos bifurcados o estrellados. Pinnas 7-11 pares por hoja, 0,9-2,5 $\times$ 0,7-13 cm, ascendentes, pinnatífidas, simétricas, el ápice redondeado, las inferiores reducidas. Segmentos 2-10 por pinna, 0,3-0,8 × 0,2 cm, márgenes ondulado-crispados. Soros 1-5 por pinna. Involucros orbiculares, $2 \times 1,5 \mathrm{~mm}$, de borde irregular a dentado, con numerosos pelos simples en el extremo, escasamente inmersos en la base. Receptáculos insertos. Esporangios 12-22 por soro.

Habita en Jamaica, México, Guatemala, Costa Rica, Venezuela, Colombia, Perú, Bolivia, Brasil (Tryon \& Stolze 1993) y en Argentina, en Jujuy y Salta (Larsen et al. 2010). Se lo encuentra en selvas húmedas, epifito o sobre el sustrato orgánico en laderas sombrías.

Observaciones. Hymenophyllum crispum se diferencia claramente de $H$. capurroi por su follaje, ondulado-crispado en el primero y plano en el segundo. Además de la densidad de pelos, mucho mayor en $H$. capurroi, éstos son biestrellados tanto en los márgenes como en las venas.

Material seleccionado: ARGENTINA, JUJUY, Depto. Ledesma, Parque Nacional Calilegua, Aguada del Tigre, 1630 m, 05.XII.2010, C. Larsen \& M. Arana 154 (SI, RCVC). SALTA, Depto. Santa Victoria, Parque Nacional Baritú, Ayo. Baritú, bosque de Podocarpus sp., 1600-1700 m, 4.X.1985, Brown 2019 (SI). BOLIVIA, LA PAZ, Prov. Murillo, Zongo Cambaya, 16.XII.1982, Lewis 82593 (LPB); Prov. Franz Tamayo, Parque Nacional Madidi, 1850-2000 m, 30.VI.2008, Fuentes et al. 4684 (LPB).

3.3. Hymenophyllum polyanthos (Sw.) Sw., J. Bot. (Schrader) 1800 (2): 102. 1802. Trichomanes polyanthos Sw., Prodr.: 137. 1788. TIPO: "Jamaica", O. P. Swartz s.n. (Holotipo S06-1597!; Isotipos B-W 20235!, BM000936765!, S-R-2978!, S-R6211 !, S-R-6212!).

Fig. 5e-g

Rizomas rastreros, parduzcos, 0,19-0,35 $\mathrm{mm}$ de diámetro, con pelos simples, septados, adpresos, ferrugíneos, laxamente dispuestos (más densos cerca de la base). Hojas 4-15 cm; pecíolos 0,7-4,8 cm, con pelos sólo en la base similares a los del rizoma, alados, parcialmente alados o no alados. Láminas 3,5-13 × 1,8-3,3 cm, ovado u oblongo a linear-lanceoladas, 2-3-pinnatífidas, glabras, la base reducida. Raquis alado, glabro. Pinnas 6-15 pares por hoja, 2-3-pinnatífidas, $0,3-1,8(2,5) \times 0,2-1,1 \mathrm{~cm}$, ovadas, ascendentes, con ramificaciones hacia ambos lados. Segmentos 0,9-1,5 $\mathrm{mm}$ de ancho, linear-oblongos, los márgenes enteros, aplanados, el ápice emarginado, con ramificación dicotómica. Soros 3-21 por pinna. Involucros 1,3-1,9 × 0,9-1,6 mm, elípticos, oblongos u ovados, ovoides, la base obtusa a ligeramente cuneada, el ápice redondeado a ligeramente agudo, a veces emarginado o premorso. Receptáculos insertos. Esporangios 6-15 por soro.

Especie pantropical (Larsen et al. 2010), en Sudamérica se lo encuentra en selvas húmedas, como epífito de helechos arborescentes o saxícola, también sobre troncos caídos.

Observaciones. Es uno de los helechos tropicales más ampliamente distribuido (Larsen et al. 2010), y requiere una revisión para confirmar su identidad, ya que probablemente constituya un complejo de especies. En Argentina se lo puede encontrar creciendo junto con $H$. crispum, del que se diferencia por el margen de la hoja entero y plano, las hojas completamente glabras, y el indusio glabro, con su base inmersa en el segmento terminal.

Material seleccionado: ARGENTINA, JUJUY, Depto. Ledesma, Parque Nacional Calilegua, Río de las Piedras, 1750 m, 01.X.1986, Iudica \& Ramadori 340 (SI); cerca de Aguada del Tigre, 1630 m, 05.VII.2010, C. Larsen \& M. Arana 159 (SI, RCVC). SALTA, Depto. Santa Victoria, Parque Nacional Baritú, 1600-1700 m, 4.X.1985, A. Brown 2024 (SI); Depto. Iruya, Río Piedra, 1120 m, 17.X.1982, P.R. Legname 8735 (LIL).

\subsection{Hymenophyllum tunbrigense var. cordobense} Hieron., en Engler, Bot. Jahrb. 22: 361. 1896. TIPO: Argentina, "Córdoba. Sierra de Achala, en la quebrada, cerca del Puesto Alegre", 5-III-1877, G.H.E.W. Hieronymus 873 (Lectotipo designado por Larsen 2014: 104, CORD00001407!; Isolectotipo SI012004!).

Fig. 5a-d

Rizomas largamente rastreros, filiformes, pardo oscuros, de 0,14-0,3 mm de diámetro.; pelos del rizoma rojizos, simples, de 0,3-0,6 $\mathrm{mm}$, escasos. Hojas distantes, de 3,3-10 cm. Pecíolos capilares, pardos a pardo oscuros, de 0,5-2,5 cm long., no alados, con algunos pelos rojizos, simples paucicelulares, de 0,3-0,46 mm. Láminas de contorno ovado-lanceolado u ovado-lineal-lanceolado, pinnado-pinnatífidas a 3-4-pinnatífidas, de 2,7-9 $\times$ 0,5-1,2 cm, con pequeños pelos bicelulares rojizos sobre el raquis y las venas. Raquis pardo oscuro, alado hacia el ápice, a partir del cuarto al séptimo par de pinnas. Pinnas flabeladas a rómbicas, a veces trapeciformes, distanciadas, ascendentes, en número de 6-12 pares por lámina, con pocos segmentos (2-4), 3-4-pinnatífidas, las basales divididas hacia ambos lados, las apicales más desarrolladas en el lado acroscópico. Segmentos lineales, de 0,8-1 mm lat., con ápice redondeado y margen dentado. Soros 
subaxilares, 1 por pinna, raramente más, sobre un corto segmento angostamente alado, en ocasiones lobulado, arqueado en la base. Involucros ovados, no inmersos en la lámina, de base algo engrosada, aguda, ápice redondeado, de margen entero o dentado, valvas abiertas hasta más de la mitad. Receptáculo inserto, cilíndrico a fusiforme, grueso, con aprox. 30 esporangios.

La especie $H$. tunbrigense constituye un taxón cosmopolita que, en Argentina, está representada por dos variedades alopátricas: $H$. tunbrigense var. tunbrigense en los bosques andino-patagónicos de Argentina y Chile (Larsen et al. 2013), e $H$. tunbrigense var. cordobense, que habita la región serrana de Argentina en las provincias de Catamarca, Jujuy, Salta, Tucumán y Córdoba (Arana et al. 2013), donde crece sobre rocas con cubierta muscinal, entre 1200 y 2100 m s.m.

Observaciones. La variedad cordobense, difiere de la variedad tipo por sus láminas de contorno lanceolado, angostas (vs. contorno ovado u ovadoelíptico) y con pinnas remotas (vs. aproximadas, hasta imbricadas) (de la Sota 1977). Recientemente se analizaron diferencias morfológicas y anatómicas entre
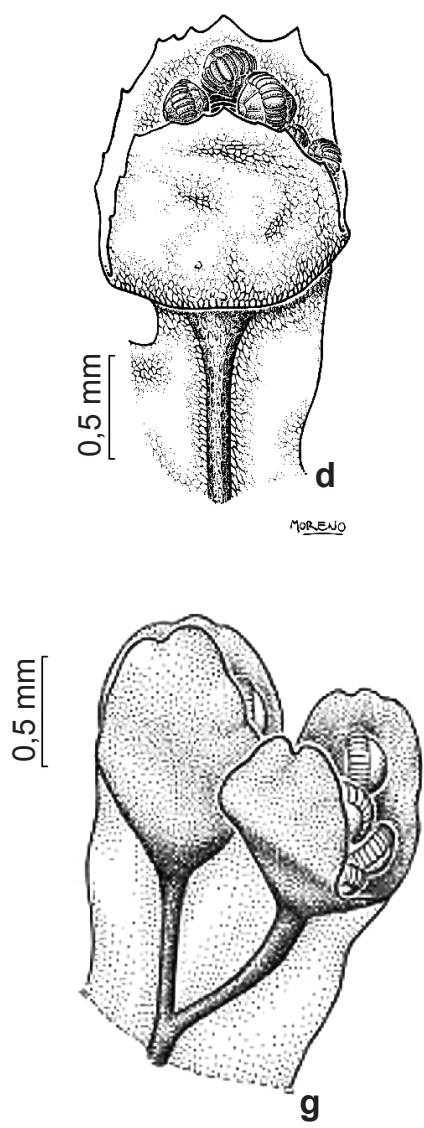
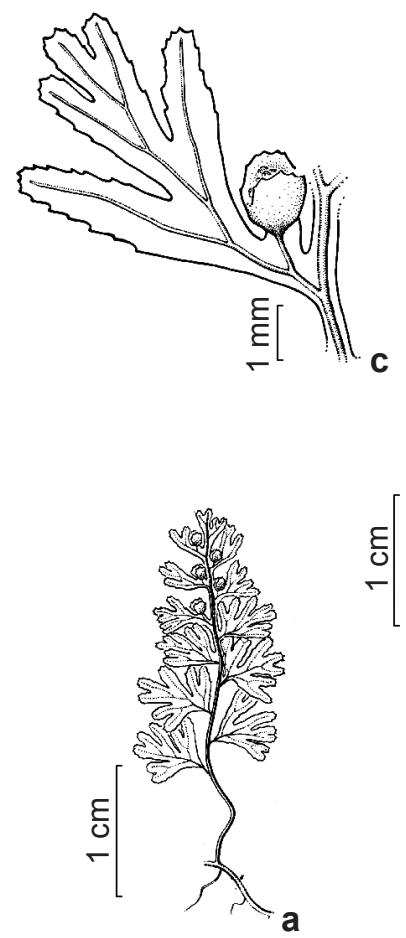
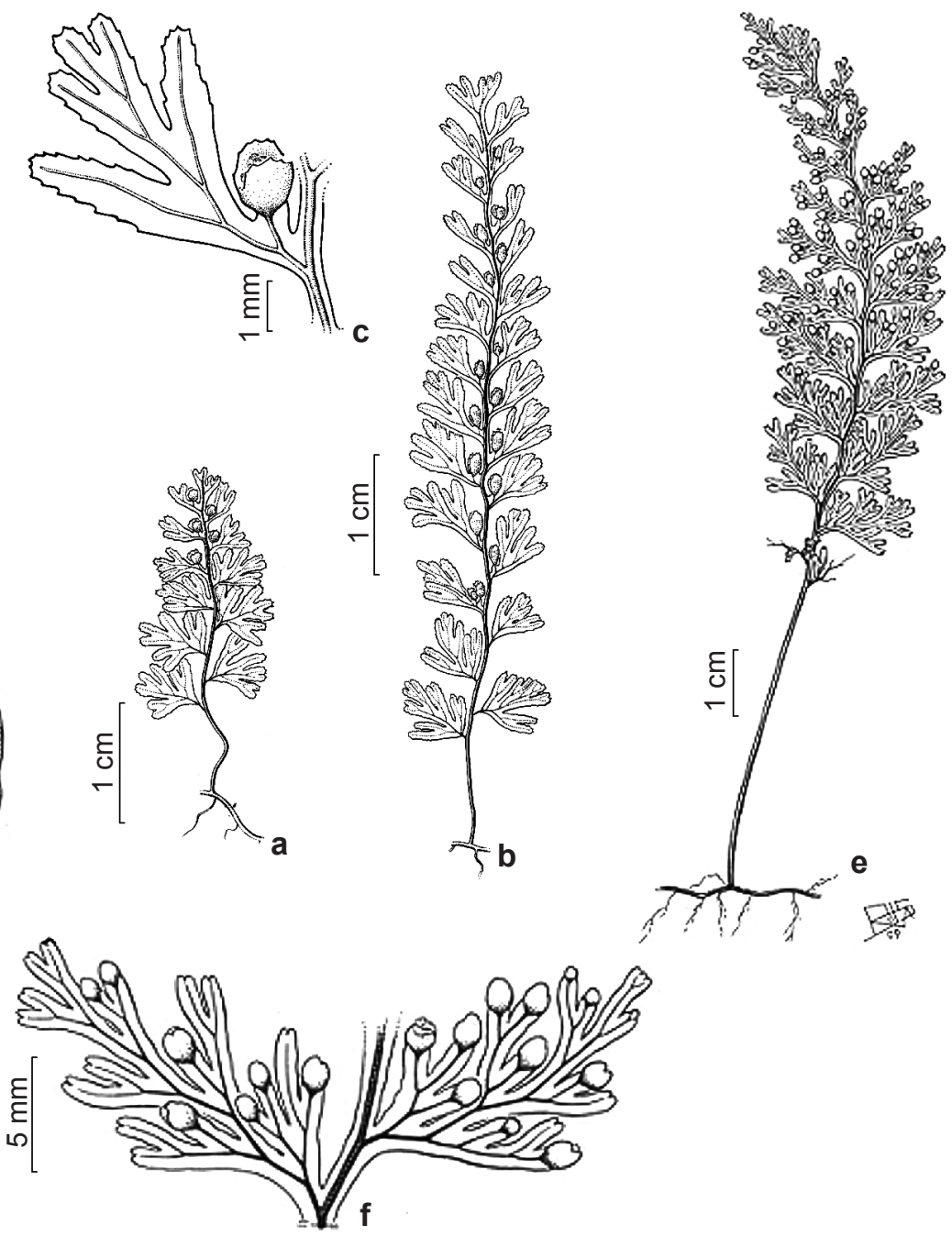

Figura 5 - a-d. Hymenophyllum tunbrigense var. cordobense - a-b. aspecto general; c. detalle de una pinna fértil; d. detalle de un soro. (C. Larsen 160 - SI). e-g. Hymenophyllum polyanthos - e. aspecto general; f. detalle de un par de pinnas; g. detalle de un par de soros. (A. Brown 2024 - SI).

Figure 5 - a-d. Hymenophyllum tunbrigense var. cordobense - a-b. habit; c. detail of a pinnae with sori; d. detail of a sorus. (C. Larsen 160 - SI). e-g. Hymenophyllum polyanthos - e. habit; f. detail of a pair of pinnae; g. detail of a pair of sori. (A. Brown 2024 - SI). 
las variedades de $H$. tunbrigense que, junto con nuevos datos moleculares y su ubicación en la filogenia de Hymenophyllum permitirían reconocer las variedades a nivel específico (Larsen et al. en prep.).

Material seleccionado: ARGENTINA. CÓRDOBA, Depto. Cruz del Eje, Ruta Provincial 28, de Tanti a Taninga, pie oriental de Los Gigantes, 1860 m, 08.X.2009, F.O. Zuloaga 11314 (SI). Depto. Calamuchita, Villa Alpina, 11/10/2009, C. Larsen 68 (SI). JUJUY, Depto. Ledesma, Parque Nacional Calilegua, Aguada del Tigre, 1630 m, 05.VII.2010, C. Larsen \& M. Arana 160 (SI, RCVC). SALTA, Depto. Capital, Quebrada de San Lorenzo, saxícola, 2100 m, 25.VIII.1985, C. Palací 149 (MCNS). Depto. Santa Victoria, Los Toldos, sobre capa muscinal en tronco, $1600 \mathrm{~m}, 6$.VII.1998, O. Martinez \& E. de la Sota 674 (MCNS). TUCUMÁN, Depto. Chicligasta, Casa de Piedra, afluente del arroyo del Bajo Grande, 1233 m, 04.XI.2009, C. Larsen \& E. Ulibarri 107 (SI).

\section{Polyphlebium angustatum (Carmich.)} Ebihara \& Dubuisson, Blumea 51(2): 240. 2006. Vandenboschia angustata (Carmich.) Copel., Philipp. J. Sci. 73: 466. 1941. Trichomanes angustatum Carmich., Trans. Linn. Soc. London 12: 513. 1819. TIPO: Tristan da Cunha, D. Carmichael s.n. (Holotipo K (?) no localizado; isotipo BM 001044269 imagen digital!).

Fig. 6f-g

Plantas con rizomas rastreros, delgados, filiformes, de aprox. 0,5 $\mathrm{mm}$ de diámetro, densamente cubierto con pelos castaños; con raíces delgadas. Hojas remotas de hasta $20 \times 5$ $\mathrm{cm}$ long.. Pecíolos delgados, glabros de hasta 5 $\times 0,3 \mathrm{~cm}$. Láminas de contorno lineal-elíptico a ovado lanceolado, simétricas a ambos lados del raquis, $2-3$ pinnadas, glabras, de $10-12 \times 2-3 \mathrm{~cm}$, abrupta o gradualmente reducida hacia la base. Raquis primario sin alas. Pinnas remotas, con raquis secundario angostamente alado. Segmentos ascendentes, no superpuestos, los del último orden lineales, de 0,5-0,8 $\mathrm{mm}$ de ancho, con una única vena y de ápice redondeado. Soros subaxilares, uno a cinco por pinna, con involucros alados, las alas de tres o cuatro hileras de células de ancho, cónicos, sin labios diferenciados. Receptáculo largamente exerto.

Especie frecuente en América tropical, desde México y el Caribe (Cuba, Puerto Rico), Isla Tristán da Cunha y Guatemala hasta Bolivia, Brasil, Uruguay y Norte de Argentina (Davidse et al. 1995), en provincias del Noroeste y en Misiones, donde se comporta como epífito en cáudices de Alsophila setosa Kaulf. (Marquez \& Yañez 2012). En las Yungas del sur habita en lugares sombríos, sobre rocas y troncos muertos en lugares hiperhúmedos en las provincias de Jujuy y Salta.

Observaciones: Polyphlebium angustatum es muy afín a $P$. capillaceum (L.) Ebihara \& Dubuisson, de la cual es muy difícil separarla. Las principales diferencias se encuentran a nivel de las costas, aladas en $P$. angustatum, mientras que en $P$. capillaceum no hay tejido expandido como alas en los ejes, o si lo hay, es solo cerca del ápice. Otras diferencias se encuentran en los últimos segmentos, que son más angostos (los de $P$. angustatum son de 0,1 a 0,4 mm de ancho). Además, se diferencian por el indusio que no es alado, o, si existen alas, éstas solo están constituidas por una hilera de células de ancho. $P$. capillaceum es una especie que habita desde México hasta Perú (Tryon \& Stolze 1993). Material seleccionado: ARGENTINA. JUJUY, Depto. Ledesma, Parque Nacional Calilegua, Aguada del Tigre, 1630 m, 05.VII.2010, C. Larsen \& M. Arana 152 (SI, RCVC). SALTA. Dpto. Orán, Aguas Blancas, finca El Arrazayal, salto 20 m río El Nogal, 1000 m, 24.III.1985, C. Palací 108 (MCNS). Depto. Santa Victoria, Parque Nacional Baritú, Arroyo Baritú, bosque de Podocarpus, 1600-1700 m, 04.X.1985, A. Brown 2020 (SI).

\section{Análisis Panbiogeográfico}

Los patrones de distribución de las nueve especies de Hymenophyllaceae de las Yungas sur (Fig. 7a-i) se componen de: 1) Un Trazo individual pantropical, correspondiente a Hymenophyllum polyanthos (Fig. 7e). En los análisis moleculares realizados con muestras de localidades distantes (Japón, Tahití, Isla Reunión, Bolivia), esta especie se ubicó muchas veces ocupando posiciones diferentes dentro del clado del subgénero Mecodium (Hennequin et al. 2010), lo cual muestra la necesidad de un estudio más exhaustivo para esclarecer su identidad; 2) Un Trazo individual gondwánico, que conecta África y Madagascar con América del Sur, perteneciente a la especie Didymoglossum reptans (Fig. 7i) Trazos individuales neotropicales, correspondientes a Didymoglossum hymenoides y D. krausii, Crepidomanes pyxidiferum, Hymenophyllum crispum y Polyphlebium angustatum. Todas estas especies establecen la conexión de la región de las yungas sur con la región de la mesopotamia argentina y austral brasileña. 4) Trazos individuales endémicos, de las especies Hymenophyllum capurroi e $H$. tunbrigense var. cordobense (Fig. $7 \mathrm{~g}, \mathrm{~h}$ ), características de las Yungas sur o Bosques Tucumano-Bolivianos, llegando la segunda hasta los sistemas serranos del centro de Argentina. 
Como resultado de la superposición de todos los trazos individuales, se observa un trazo generalizado (Fig. 8) centro y sudamericano, en donde el mayor número de componentes con distribución en las Yungas sur están en contacto con los Andes Tropicales, llegando hasta el sur de México y las Antillas por el norte, y hasta las Guayanas por el este. Además se demuestra que las Yungas sur poseen vinculaciones estrechas con el dominio Paraná, (sensu Morrone 2014), con algunas especies (Fig. 7b,c,d,f,i) salvando la barrera árida representada por el dominio Chaqueño (sensu Morrone 2014). Finalmente, sólo se obtuvo un trazo generalizado, por lo tanto no consideran nodos, que ocurren cuando existe convergencia de trazos generalizados; esto implica que en el noroeste de la Argentina, para la Familia Hymenophyllaceae, no hay confluencia de biotas ancestrales, lo que implica que todas las especies presentes corresponden a un linaje con una historia geobiótica común.
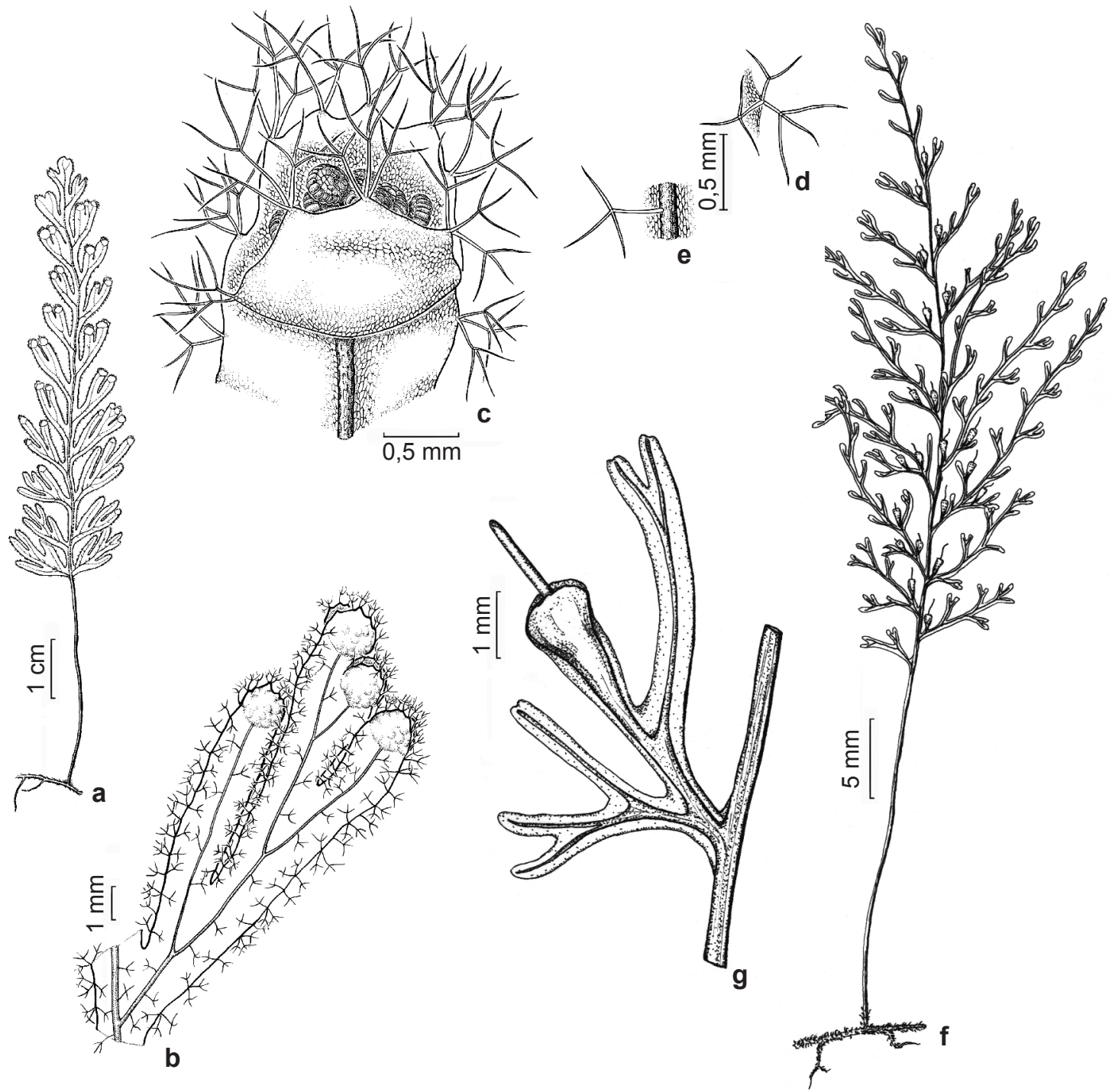

Figura 6 - a-e. Hymenophyllum capurroi - a. aspecto general; b. detalle de una pinna; c. detalle de un involucro; d. detalle de un pelo del margen de la lámina; e. detalle de un pelo del raquis. (C. Larsen 138 - SI). f-g. Polyphlebium angustatum - f. aspecto general; g. detalle de un segmento fértil. (modificado de E.R. de la Sota 1977).

Figure 6 - a-e. Hymenophyllum capurroi - a. habit; b. detail of a pinnae; c. detail of an involucre; d. detail of a hair from lamina margin; e. detail of a hair from the rachis. (C. Larsen 138 - SI). f-g. Polyphlebium angustatum - f. habit; g. detail of a fertile segment. (modificado de E.R. de la Sota 1977). 
De acuerdo a los trazos, es posible establecer tres patrones distribucionales principales: 1) Gondwánico: con el trazo individual de D. reptans (Fig. 7i), ya establecido previamente por Arana et al. $(2011,2012)$ para las licofitas y por Moran \& Smith (2001) y Janssen et al. (2007) para diversos grupos de helechos; 2) Pantropical: correspondiente al trazo individual de $H$. polyanthos, que conecta el área gondwánica América del Sur-ÁfricaMadagascar con las islas del sudeste asiático (Fig. 7e). Estas amplias distribuciones, en trabajos previos han sido explicadas por dispersión a larga distancia (Prado \& Hirai 2014), debido a que los helechos poseen esporas muy pequeñas y livianas que pueden encontrarse a gran altura en la atmósfera, aunque las esporas que llegan a gran altitud están irreversiblemente dañadas por los rayos ultravioletas (Schaeffer 2011); y en el caso particular de las esporas de Hymenophyllaceae, al contener clorofila, poseen un período de viabilidad muy corto. Además las Hymenophyllaceae, por su alta dependencia de la humedad ambiental, no sobrepasarían barreras biogeográficas como pueden ser los océanos, las montañas y, en particular, las regiones áridas (Hennequin et al. 2006) representada en la región por la Zona de Transición Sudamericana. De acuerdo al enfoque Panbiogeográfico, los eventos tectónicos y climáticos son la mayor fuerza de los cambios geográficos y fragmentación de los componentes bióticos ancestrales (Echeverry \& Morrone 2013). Hennequin et al. (2006) han demostrado que Hymenophyllum posee una distribución ancestral gondwánica, y que luego sufrió eventos vicariantes que se corresponden a la secuencia de fragmentación del supercontinente Gondwana en cuatro regiones: América del Sur, África, Madagascar-India y la Antártida-Australia-Nueva Zelanda-Nueva Caledonia. Teniendo en cuenta el trazo individual de $H$. polyanthos, la conexión con Malasia-Polinesia se podría explicar por eventos dispersivos (= de movilidad) desde regiones gondwánicas próximas a través de Papua- Nueva Guinea (Hennequin et al. 2006).

Por último el patrón 3) Neotropical: conformado por el resto de las especies (Didymoglossum hymenoides y D. krausii, Crepidomanes pyxidiferum, Hymenophyllum crispum y Polyphlebium angustatum), resultado del evento vicariante gondwánico y que contribuye a la hipótesis del componente biótico ancestral encontrado previamente en licofitas y otros grupos de helechos (Arana et al. 2012, 2013), en donde los Andes Centrales y las Sierras Subandinas están vinculadas estrechamente con los Andes del Norte (Gregory-Wodzicki 2000), tal cual fue propuesto por de la Sota (1973) y Ponce et al. (2002) y en consonancia con el denominado "patrón distribucional montano" por Ferro \& Morrone (2014). Aquí también se establece una conexión entre las selvas Paranaense y Yungueñas mediante las especies Hymenophyllum crispum, Polyphlebium angustatum, Didymoglossum hymenoides y D. krausii (Fig. 7b,c,d,f, y i). Este patrón es compartido por otras plantas: las licofitas Selaginella sellowii Hieron. y Diphasiastrum thyoides (Humb. ex Bonpl. \& Willd.) Holub (Arana et al. 2012); helechos del género Asplenium L. (Ganem et al. 2007), Dennstaedtia Bernh. (Yañez et al. 2014), Osmunda (Arana \& Ponce 2015), numerosas angiospermas (Pennington et al. 2000; Garcia et al. 2014) y por grupos animales, como las aves que aprovechan los bosques en galería a la largo de los cursos de agua de los ríos Bermejo, Pilcomayo, Paraguay y Paraná (Nores 1992). Estos bosques en galería constituirían relictos de una distribución más amplia de las selvas durante los períodos interglaciares del Pleistoceno y Holoceno, en donde las selvas de las Yungas y Paranaense cubrirían un territorio mucho mayor que el actual (Van der Hammen 1974; Bigarella \& de AndradeLima 1982; Pennington et al. 2000). Esta gran superficie boscosa fue gradualmente fragmentada en dos partes, una en el oeste (Yungas) y otra en el este (Paranaense) por el avance de los bosques xerófilos del domino Chaqueño, provocando la consecuente vicarianza dinámica de los taxones que componen estos bosques húmedos subtropicales, que ocupan dos áreas disyuntas entre los $23^{\circ} \mathrm{S}$ y $29^{\circ} \mathrm{S}$ (Morrone \& Coscarón 1996; Pennington et al. 2000; Katinas \& Crisci 2008). Esta secuencia de eventos está sustentada, además, por evidencias paleontológicas, paleoclimatológicas y geológicas que indican que un clima templado prevaleció en Sudamérica durante el Terciario y fue seguido de un enfriamiento y aridificación en el Oligoceno y Mioceno, proceso completado por el alzamiento de los Andes (Romo \& Morrone 2011; Simões et al. 2012). La región de las Yungas se caracteriza por un fuerte gradiente altitudinal que se refleja en la composición específica de la vegetación, dependiendo de este gradiente se encuentran especies adaptadas a las más diversas condiciones ambientales (sequía, altas temperaturas, elevados niveles de humedad, heladas y nevadas invernales). Esta situación genera condiciones propicias para 


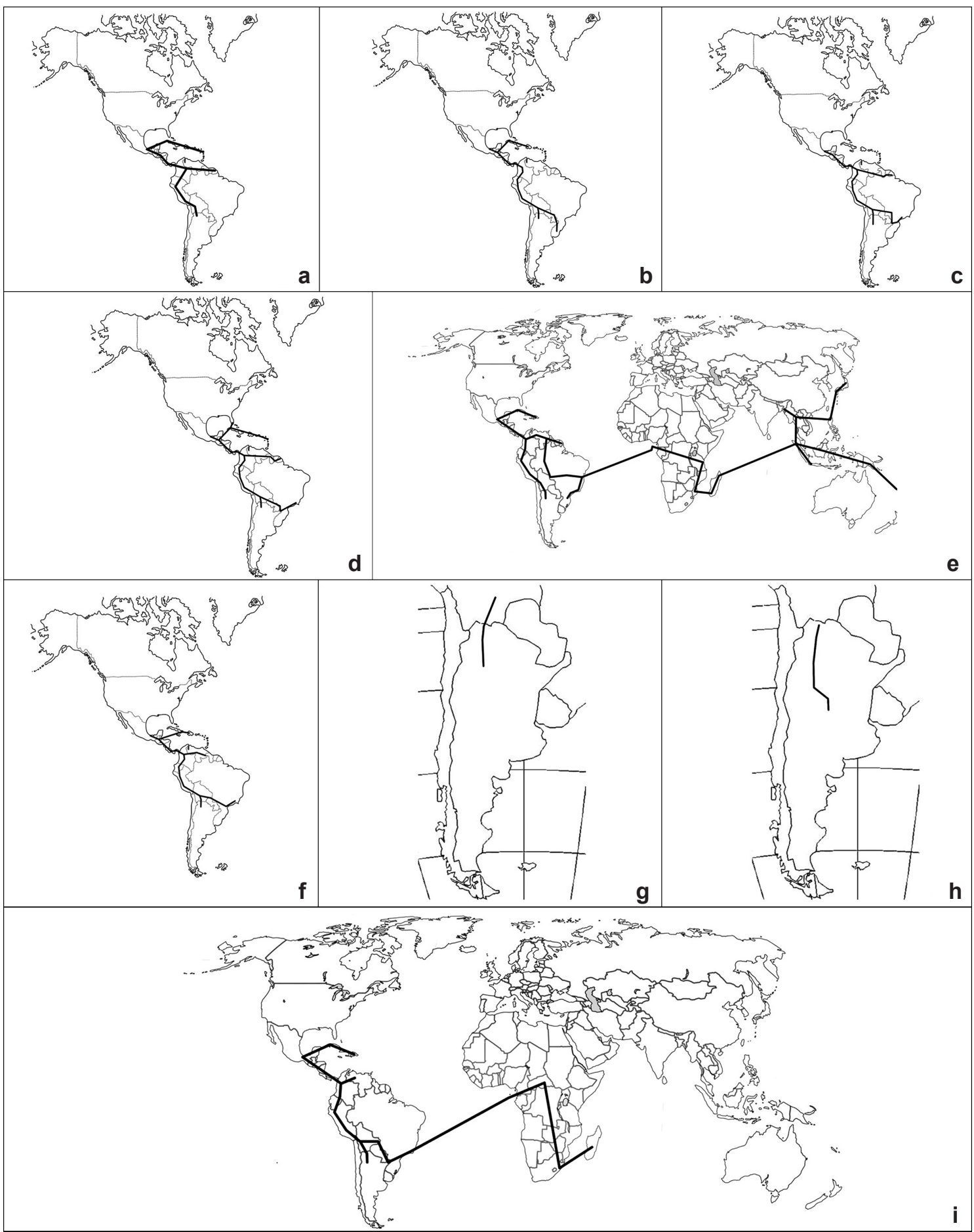

Figura 7-a-i. Trazos individuales de las especies de Hymenophyllaceae presentes en las Yungas sur. a. Crepidomanes pyxidiferum; b. Polyphlebium angustatum; c. Didymoglossum hymenoides; d. D. kraussii; e. Hymenophyllum polyanthos; f. Hymenophyllum crispum; g. H. capurroi; h. H. tunbrigense var. cordobense; i. Didymoglossum reptans.

Figure 7 - a-i. Individual Tracks of the species of Hymenophyllaceae from southern Yungas. a. Crepidomanes pyxidiferum; b. Polyphlebium angustatum; c. Didymoglossum hymenoides; d. D. kraussii; e. Hymenophyllum polyanthos; f. Hymenophyllum crispum; g. H. capurroi; h. H. tunbrigense var. cordobense; i. Didymoglossum reptans. 


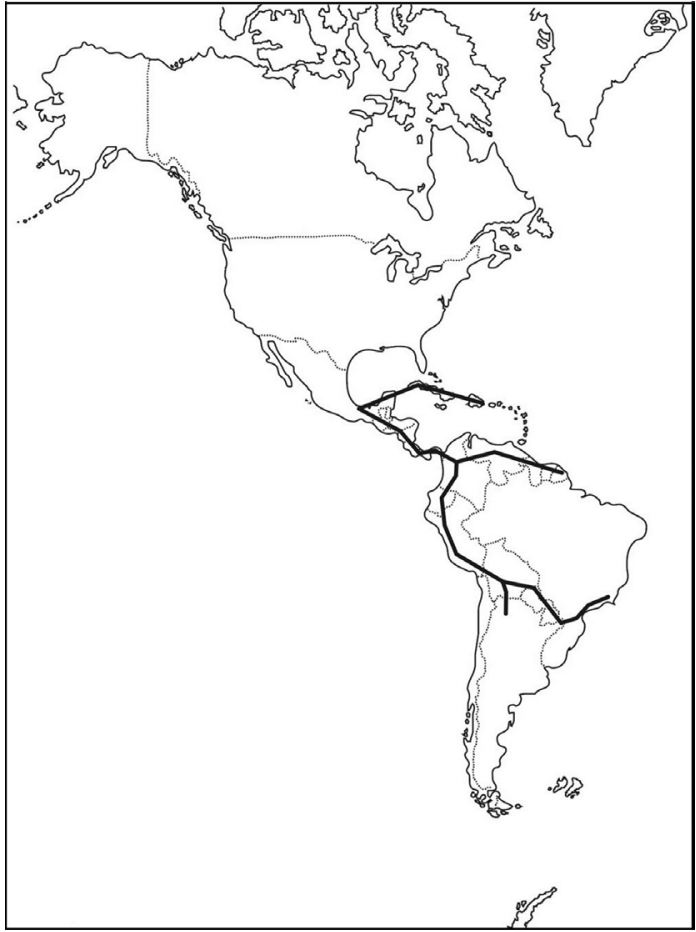

Figura 8 - Trazo generalizado de las especies de Hymenophyllaceae.

Figure 8-Generalized track of the species of Hymenophyllaceae.

la coexistencia de especies de diferentes orígenes biogeográficos a lo largo del gradiente altitudinal (Brown et al. 2006). Las Hymenophyllaceae de esta región, en donde conviven especies con una distribución pantropical $(H$. polyanthos y $D$. reptans) y otras exclusivamente neotropicales (Hymenophyllum crispum, Polyphlebium angustatum, Didymoglossum hymenoides y D. krausii) ocupan los ambientes hiperhúmedos de este gradiente.

En general se postula que la distribución actual de Hymenophyllaceae se originó por eventos vicariantes que fragmentaron una distribución generalizada gondwánica ancestral y que luego se complejiza por eventos de movilidad (=dispersión) a sectores cercanos con extinciones en diversas áreas (Copeland 1947; Farrar 1993; Hennequin 2003). Aunque esto adolece de evidencia fósil (Dubuisson et al. 2013), es posible inferir; a partir de la filogenia de la familia, donde linajes de posición basal poseen una distribución intercontinental en el hemisferio sur (por ejemplo en los subgéneros Fuciformia, Globosa, Hymenoglossum del género Hymenophyllum), mientras que linajes que reúnen la presencia de caracteres derivados (subgéneros Mecodium, Hymenophyllum, Sphaerocionium del mismo género) presentan una distribución pantropical (Hennequin 2004); que la familia, a partir de una distribución austral gondwánica se diversificó posteriormente en áreas tropicales (Iwatsuki 1979; Hennequin 2004) como por ejemplo, las Yungas. Un proceso vicariante similar ha sido demostrado también para Cyatheaceae (Korall \& Pryer 2014).

La zona austral de la Argentina y Chile, que constituye uno de los centros de concentración de especies de Hymenophyllum (Larsen et al. 2013), pertenece a la región Andina (sensu Morrone 2015) y está separada de la región Neotropical por la Zona de Transición Sudamericana. Ésta última comprende las provincias biogeográficas del Páramo Norandino, Desierto Costero Peruano, Puna, Atacama, Prepuna y Monte (Morrone 2015), todos ellos ambientes áridos, sin bosques; estas regiones constituirían una barrera vicariante exitosa para la distribución de Hymenophyllaceae, ya que no hay ninguna vinculación entre la biota de la región Andina con la biota ancestral Neotropical establecida para las Yungas australes.

\section{Conclusiones}

En el noroeste de la Argentina y el sur de Bolivia se encuentra el límite austral de las pluviselvas andinas de América del Sur, llamadas "Selva tucumano-oranense", "Bosques Tucumano-Bolivianos" o Yungas australes, donde se encuentran representados cuatro géneros de Hymenophyllaceae y nueve especies: Crepidomanes $(C$. pyxidiferum), que se registra por primera vez para la Argentina, Didymoglossum (3) Hymenophyllum, (4) y Polyphlebium ( $P$. angustatum).

Las particularidades microambientales de las Yungas generan condiciones propicias para la coexistencia de especies de Hymenophyllaceae con diferentes orígenes biogeográficos a lo largo del gradiente altitudinal, en donde conviven especies con una distribución pantropical (H. polyanthos y $D$. reptans), otros exclusivamente neotropicales (Hymenophyllum crispum, Polyphlebium angustatum, Didymoglossum hymenoides y D. krausii) y taxones exclusivos de esta región, como Hymenophyllum capurroi y H. tunbrigense var. cordobense con su límite de distribución austral en las Sierras Pampeanas del centro de Argentina, donde es el único representante de esta familia, lo que apoya la vinculación entre las Sierras Subandinas y las del centro argentino. Por otra parte se establece una conexión entre 
las biotas de Hymenophyllaceae presentes en las Yungas y la selva Paranaense mediante las especies Hymenophyllum crispum, Polyphlebium angustatum, Didymoglossum hymenoides y D. krausii; salvando la diagonal árida formada por el dominio Chaqueño, que ocupa el norte y centro de la Argentina, sur de Bolivia, oeste y centro del Paraguay, y centro y noroeste del Brasil. Este patrón es compartido por diferentes linajes, tanto de plantas como de animales y contribuye con el proceso de fragmentación de los bosques sudamericanos debido a la aparición de la cuña árida chaqueña. Por otra parte, la Zona de Transición Sudamericana, conformada por ambientes áridos y semi-áridos, representa una barrera geobiótica exitosa para el componente biótico de Hymenophyllaceae de las Yungas argentinas.

\section{Agradecimientos}

A los dibujantes Francisco Rojas, Marcelo Moreno, M. del C. Otero, Heraldo Mussolini, y especialmente a Elías de la Sota "in memoriam", por las ilustraciones de su Flora de Pteridofitas de Jujuy. Al personal de los herbarios visitados y consultados, su amable atención y envío de materiales. Al personal de los Parques Nacionales de Calilegua y Baritú, el apoyo logístico brindado durante nuestras estadías. A los compañeros de campañas y viajes de campo Antonia Oggero, Lujan Luna y Alejandra Ganem. Al CONICET, las becas y subsidios otorgados. A Marli Pires Morim, Simone Bittencourt, Claudine Mynssen y dos revisores anónimos cuyos comentarios y sugerencias mejoraron sensiblemente el manuscrito. $\mathrm{Y}$ al Instituto de Botánica Darwinion y la Universidad Nacional de Río Cuarto, los permisos y la utilización de los laboratorios e instalaciones. Este trabajo se realizó en el marco del proyecto Flora Argentina ( $<$ http://www.floraargentina.edu.ar $>)$, financiado por el PIP 537-CONICET.

\section{Referencias}

Ambrose, A.R. 2004. Water-holding capacity of canopy soil mats and effects on microclimates in an old-growth redwood forest: A report to Savethe-Redwoods League. Master in Science Thesis. Humboldt State University, Arcata. 95p.

Arana, M.D.; Morrone, J.J.; Ganem, M.A.; Luna, M.L.; Ramos Giacosa, J.P. \& Giudice, G. 2012. Diversidad y análisis panbiogeográfico de las licofitas (Embryopsida: Lycopodiidae) del Parque Nacional Calilegua, Jujuy, Argentina. Iheringia (Série Botanica) 67: 177-188.
Arana, M.D.; Morrone, J.J.; Ponce, M.M. \& Oggero, A.J. 2013. Patrones biogeográficos de los helechos de las sierras de Córdoba (Argentina) y sus implicancias en la conservación. Gayana Botánica 70: 357-376.

Arana, M.D. \& M.M. Ponce. 2015. Osmundaceae en Argentina, Paraguay y Uruguay. Darwiniana, nueva serie 3: 27-37.

Arcand, N.N. \& Ranker, T.A. 2008. Conservation biology. In: Ranker, T.A. \& C.H. Haufler (eds.). The Biology and evolution of ferns and Lycophytes. Cambridge University Press, New York. Pp: 257-283.

Bigarella, J.J. \& de Andrade-Lima, D. 1982. Paleoenvironmental changes in Brazil In: Prance, G.T. (ed.). Biological diversification in the tropics. Columbia University Press, New York. Pp. 27-40.

Brown, A.D.; Grau, H.R.; Malizia, L. \& Grau, A. 2001. Los Bosques Nublados de la Argentina. In: Kappelle, M. y Brown, A.D. (eds.). Bosques Nublados de Latinoamerica. Editorial INBio, Costa Rica. Pp: 623-659.

Brown A.; Grau, H.; Lomáscolo, T. \& Gasparri, N. 2002. Una estrategia de conservación para las selvas subtropicales de montaña (Yungas) de Argentina. Ecotrópicos 15: 147-159.

Brown, A.D.; Pacheco, S.; Lomáscolo, T. \& Malizia, L. 2006. Situación ambiental en los bosques andinos yungueños. In: Brown, A.; Martinez Ortiz, U.; Acerbi, M. \& Corcuera, J. (eds.). La situación ambiental Argentina 2005. Fundación Vida Silvestre Argentina, Buenos Aires. Pp: 52-71.

Cabrera, A.L. \& Willink, A. 1980. Biogeografía de América Latina. Monografía 13. Serie de Biologia. OEA, Washington, D.C. 120p.

Castellanos, A. \& Perez-Moreau, R.A. 1944. Los tipos de vegetación de la Republica Argentina. Monografías del Instituto de Estudios Geográficos, Universidad Nacional de Tucumán 4: 1-154.

Copeland, E.B. 1947. Genera Filicum, Chronica Botanica. Waltham, Massachusetts. 247p.

Davidse, G.; Sousa Sánchez, M. \& Knapp, S. 1995. Psilotaceae a Salviniaceae, In: Davidse, G.; Sousa Sánchez, M. \& Chater, A.O. (eds.). Flora Mesoamericana. Universidad Nacional Autónoma de México, México-D.F. Pp. i-xxi, 1-470.

de la Sota, E.R. 1973. La distribución geográfica de las pteridófitas en el cono sur de América meridional. Boletín de la Sociedad Argentina de Botánica 15: 23-34.

de la Sota, E.R. 1977. Pteridophyta. In: Cabrera, A.L. (dir.). Flora de la Provincia de Jujuy. Colección Científica del Instituto Nacional de Tecnología Agropecuaria 13: XIV + 275.

Dubuisson, J.Y.; Hennequin, S.; Rakotondrainibe, F. \& Schneider, H. 2003a. Ecological diversity and adaptive tendencies in the tropical fern 
Trichomanes L. (Hymenophyllaceae) with special reference to climbing and epiphytic habits. Botanical Journal of the Linnean Society 142: 41-63.

Dubuisson, J.Y.; Hennequin, S.; Douzery, E.J.P.; Cranfill, R.B.; Smith, A.R. \& Pryer, K.M. 2003 b. $r b c \mathrm{~L}$ phylogeny of the fern genus Trichomanes (Hymenophyllaceae), with special reference to neotropical taxa. International Journal of Plant Sciences 164: 753-761.

Dubuisson, J.-Y.; Rouhan, G.; Gralla, A.; Hennequin, S.; Senterre, B.; Pynee, K. \& Ebihara, A. 2013. New insights into the systematics and evolution of the filmy fern genus Crepidomanes (Hymenophyllaceae) in the Mascarene Archipelago with a focus on dwarf species. Acta Botanica Gallica: Botany Letters 160: 173-194.

Ebihara, A.; Dubuisson, J.Y.; Iwatsuki, K; Hennequin, S. \& Motomi, I. 2006. A taxonomic revision of Hymenophyllaceae. Blumea 51: 1-60.

Ebihara, A.; Hennequin, S.; Iwatsuki, K.; Bostock, P.D.; Matsumoto, S.; Jaman, R.; Dubuisson, J.Y. \& Ito, M. 2004. Polyphyletic origin of Microtrichomanes (Prantl) Copel. (Hymenophyllaceae), with a revision of the species. Taxon 53: 935-948.

Echeverry, A. \& Morrone, J.J. 2013. Generalized tracks, area cladograms and tectonics in the Caribbean. Journal of Biogeography 40: 1619-1637.

Espinosa, D.; Morrone, J.J.; Llorente, J. \& Flores, O. 2002. Introducción al análisis de patrones en biogeografía histórica. Las Prensas de Ciencias, Facultad de Ciencias, UNAM, México-D.F. 133p.

Farrar, D.R. 1993. Hymenophyllaceae Link: Filmy Fern Family. In: Flora of North America Editorial Committee (eds.). Flora of North America North of Mexico, Pteridophytes and Gymnosperms 2: 191-197.

Ferro, I. \& Morrone, J.J. 2014. Biogeographical transition zones: a search for conceptual synthesis. Biological Journal of the Linnean Society 113: 1-12.

Ganem, M.A; Giudice, G.E.; Luna, M.L. \& de la Sota E.R. 2007. Revisión del grupo "Asplenium squamosum" en América. Candollea 62: 149-156.

Ganem, M.A.; Ramos Giacosa J.P.; Luna M.L.; Arana M.D.; Rotman A.; Ahumada O.; de la Sota E.R. \& Giudice, G.E. 2013. Diversidad de helechos y licofitas del Parque Nacional Calilegua, Provincia de Jujuy, Argentina. Boletín de la Sociedad Argentina de Botánica 48: 567-584.

García, M.V.; Prinz, K.; Barrandeguy, M.E.; Miretti, M. \& Finkeldey, R. 2014. A unifying study of phenotypic and molecular genetic variability in natural populations of Anadenanthera colubrina var. cebil from Yungas and Paranaense biogeographic provinces in Argentina. Journal of Genetics 93: 1-10.

Grau, A. \& Brown, A.D. 2000. Development threats to biodiversity and opportunities for conservation in the mountain ranges of the Upper Bermejo River
Basin, NW Argentina and SW Bolivia. Ambio 29: 445-450.

Gregory-Wodzicki, K. 2000. Uplift history of the Central and Northern Andes: A review. GSA Bulletin 112: 1091-1105.

Hennequin, S. 2003. Phylogenetic relationships within the fern genus Hymenophyllum s.l. (Hymenophyllaceae, Filicopsida): contribution of morphology and cytology. Comptes Rendus Biologies 326: 599-611.

Hauman, L. 1931. Equisse phytogéographique de l'Argentine subtropical et de ses relations avec la Géobotanique sudaméricaine. Bulletin de la Société Botanique de Belgique 64: 20-64.

Hennequin, S. 2004. Le genre Hymenophyllum Sm. (Hymenophyllaceae, Filicopsida): systématique phylogénétique, évolution morphologique et histoire biogéographique. Thèse de doctorat de l'Université Pierre et Marie Curie, París. 266p.

Hennequin, S.; Ebihara, A.; Ito, M.; Iwatsuki, K. \& Dubuisson, J.-Y. 2006. Phylogenetic systematics and evolution of the genus Hymenophyllum (Hymenophyllaceae: Pteridophyta). Fern Gazette 17: 247-257.

Hennequin, S.; Ebihara, A.; Dubuisson J.-Y. \& Schneider, H. 2010. Chromosome number evolution in Hymenophyllum (Hymenophyllaceae), with special reference to the subgenus Hymenophyllum. Molecular Phylogenetic and Evolution 55: 47-59.

Hill, J.D. \& Silander-Jr, J.A. 2001. Distribution and dynamics of two ferns: Dennstaedtia punctilobula (Dennstaedtiaceae) and Thelypteris noveboracensis (Thelypteridaceae) in a northeast mixed hardwoodshemlock forest. American Journal of Botany 88: 894-902.

Iwatsuki, K. 1979. Distribution of the filmy ferns in Palaeotropics. In: Larsen, K. \& Holm-Nielsen, L.B. (eds.). Tropical Botany, Academic Press, London. Pp. 309-314.

Janssen, T.; Kreier, H.-P. \& Schneider, H. 2007. Origin and diversification of African ferns with special emphasis on Polypodiaceae. Brittonia 59: 159-181.

Katinas, L. \& Crisci, J. 2008. Reconstructing the biogeographical history of two plant genera with different dispersion capabilities. Journal of Biogeography 35: 1374-1384.

Kenrick, P. \& Crane, P.R. 1997. The Origin and Early Diversification of Land Plants. Smithsonian Institution Press, Washington-D.C. 441p.

Kessler, M. 2010. Biogeography of ferns. In: Mehltreter, K.; Walker, L.R. \& Sharpe, J.M. (eds.). Fern Ecology. Cambridge University Press, EEUU.Pp: 22-60.

Kessler, M. \& Beck, S. 2001. Bolivia. In: Kappelle M. \& Brown, A.D. (eds.). Bosques Nublados de Latinoamerica. Editorial INBio, Costa Rica. Pp: 581-62 
Korall, P. \& Pryer, K.M. 2014. Global biogeography of scaly tree ferns (Cyatheaceae): evidence for Gondwanan vicariance and limited transoceanic dispersal. Journal of Biogeography 41: 402-413.

Larsen, C.; Martinez, O.G. \& Ponce, M.M. 2010. Nuevos registros en helechos para el Noroeste de la Argentina. Darwiniana 48: 100-108.

Larsen, C.; Ponce, M.M. \& Scataglini, M.A. 2013. Revisión de las especies de Hymenophyllum (Hymenophyllaceae) del sur de Argentina y Chile. Gayana Botanica 70: 274-329.

Marquez, G.J. \& Yañez, A. 2012. Helechos epífitos de Alsophila setosa (Cyatheaceae, Pteridophyta) en la provincia de Misiones, Argentina. Boletín de la Sociedad Argentina de Botánica 47: 435-442.

Moran, R.C. 2008. Diversity, biogeography and floristics. In: Ranker, T.A. \& Haufler, C.H. (eds.). The Biology and evolution of ferns and Lycophytes. Cambridge University Press, New York. Pp: 367-394.

Moran, R.C. \& Smith, A.R. 2001. Phytogeographic relationships between Neotropical and AfricanMadagascan pteridophytes. Brittonia 53: 304-351.

Morrone, J.J. 2004. Panbiogeografía, componentes bióticos y zonas de transición. Revista Brasileira de Entomología 48: 149-162.

Morrone, J.J. 2009. Evolutionary biogeography: An integrative approach with case studies. Columbia University Press, Nueva York. 301p.

Morrone, J.J. 2014. Biogeographical regionalisation of the Neotropical region. Zootaxa 3782: 1-110.

Morrone, J.J. 2015. Biogeographical regionalisation of the Andean region. Zootaxa 3936: 207-236.

Morrone, J.J. \& Coscarón, M.C. 1996. Distributional patterns of the American Peiratinae (Heteroptera: Reduviidae). Zoologische Medelingen, Leiden 70: 1-15.

Morrone, J.J. \& Crisci, J.V. 1995. Historical Biogeography: introduction to methods. Annual Review of Ecology and Systematics 26: 373-401.

Navarro, G. \& Maldonado, M. 2002. Geografía Ecológica de Bolivia. Centro de Ecología Simón I. PatiñoDepartamento de Difusión. Cochabamba, Bolivia. $719 p$.

Nores, M. 1992. Bird speciation in subtropical South America in relation to forest expansion and retraction. The Auk 109: 346-357.

Paciencia, M.L.B. \& Prado, J. 2005. Effects of forest fragmentation on pteridophyte diversity in a tropical rain forest in Brazil. Plant Ecology 180: 87-104.

Parris, B. 2001. Circum-Antarctic continental distribution patterns in pteridophyte species. Brittonia 53: 270-283.

Parodi, L.R. 1945. Las regiones fitogeográficas argentinas y sus relaciones con la industria forestal. In: Verdoorn, F. (ed.). Plants and plant science in Latin America. Waltham, Massachusetts. Pp. 127-132.
Pennington, R.T.; Prado, D.E. \& Pendry, C.A. 2000. Neotropical seasonally dry forests and Quaternary vegetation changes. Journal of Biogeography 27: 261-273.

Ponce, M.M. \& de la Sota, E.R. 2008. Hymenophyllaceae. In: Zuloaga, F.O.; Morrone, O.; Belgrano, M.J.; Marticorena, C. \& Marchesi, E. (eds.). 2008. Catálogo de las plantas vasculares del Cono Sur. Monographs in Systematic Botany from the Missouri Botanical Garden 107: 62-75.

Ponce, M.M.; Mehltreter, K. \& de la Sota, E.R. 2002. Análisis biogeográfico de la diversidad pteridofítica en Argentina y Chile continental. Revista Chilena de Historia Natural 75: 703-717.

Prado, J. \& Hirai, Y. 2014. Biogeography of the Brazilian atlantic forest: evidence from phylogenetic data sets and perspectives for fern and lycophyte studies. Fern Gazette 19: 241-257.

Pryer, K.M.; Schneider, H.; Smith, A.R.; Cranfill, R.; Wolf, P.G.; Hunt, J.S. \& Sipes, S.D. 2001 a. Horsetails and ferns are a monophyletic group and the closest living relatives to seed plants. Nature 409: 618-622.

Pryer, K.M.; Smith, A.R.; Hunt, J.S. \& Dubuisson, J.-Y. 2001b. $r b c \mathrm{~L}$ data reveal two monophyletic groups of filmy ferns (Filicopsida: Hymenophyllaceae). American Journal of Botany 88: 1118-1130.

Pryer, K.M.; Schuettpelz, E.; Wolf, P.G.; Schneider, H.; Smith, A.R. \& Cranfill, R. 2004. Phylogeny and evolution of ferns (monilophytes) with a focus on the early leptosporangiate divergences. American Journal of Botany 91: 1582-1598.

Romo, A. \& Morrone, J.J. 2011. Track analysis of the Neotropical Entimini (Coleoptera: Curculionidae: Entiminae). Revista Brasileira de Entomologia 55: 313-316.

Sanginés-Franco C; Luna-Vega I.; Alcántara Ayala O. \& Contreras-Medina, R. 2011. Distributional Patterns and Biogeographic Analysis of Ferns in the Sierra Madre Oriental, Mexico. American Fern Journal 101: 81-104.

Schaeffer, H. 2011. Dispersal limitation or habitat qualitywhat shapes the distribution ranges of ferns? In: Fontaneto, D. (ed.). Biogeography of Microscopic Organisms: Is Everything Small Everywhere?. Cambridge University Press, New York. Pp 234-243.

Simões, F.L.; Augusto Ferrari. A. \& Grazia, J.. 2012. Is Elsiella Froeschner, 1981 a valid genus? (Hemiptera: Heteroptera: Pentatomidae: Pentatominae). Zootaxa 3238: 39-48.

Skog, J.E. 2001. Biogeography of Mesozoic leptosporangiate ferns related to extant ferns. Brittonia 53: 236-269.

Smith, A.R.; Pryer, K.M.; Schuettpelz, E.; Korall, P.; Schneider, H. \& Wolf, P.G. 2006. A classification for extant ferns. Taxon 55: 705-731. 
Talonia, C.M. \& Escalante, T. 2013. Los nodos: El aporte de la panbiogeografía al entendimiento de la biodiversidad. Biogeografía 6: 30-42.

Thiers, B. [continuously updated]. Index Herbariorum: a global directory of public herbaria and associated staff. New York Botanical Garden's Virtual Herbarium. Available at $<$ http://sweetgum.nybg. org/ih/>. Access julio 2015.

Tryon, R.M. \& Stolze, R.G. 1989. Pteridophyta of Peru. Part I: 1.Ophioglossaceae- 12.Cyatheaceae. Fieldiana Botany new series 20: 1-145.

Van der Hammen, T. 1974. The Pleistocene changes of vegetation and climate in tropical South America. Journal of Biogeography 1: 3-26.

Wessels Boer, J.G. 1962. The new world species of Trichomanes sect. Didymoglossum and
Microgonium. Acta Botanica Neerlandica 11: 277-330.

Wolf, P.G.; Schneider, H. \& Ranker, T.A. 2001. Geographic distributions of homosporous ferns: does dispersal obscure evidence of vicariance? Journal of Biogeography 28: 263-270.

Yañez,A.;Arana, M.D.; Marquez, G.J. \& Oggero, A. 2014. The genus Dennstaedtia Bernh. (Dennstaedtiaceae) in Argentina. Phytotaxa 174: 069-081.

Zenteno-Ruiz, F.S. \& López, R.P. 2010. Composición, estructura y patrón espacial de un bosque tucumanoboliviano en el departamento de Tarija (Bolivia). Darwiniana 48: 32-44.

Zunino, M. \& Zullini, A. 2003. Biogeografía: la dimensión espacial de la evolución. Fondo de Cultura Económica, México-D.F. 359p. 
